\title{
Absolute Summability of the Trace Relation for Certain Schrödinger Operators
}

\author{
F. Gesztesy ${ }^{1}$, H. Holden ${ }^{2}$, B. Simon ${ }^{3}$ \\ ${ }^{1}$ Department of Mathematics, University of Missouri, Columbia, MO 65211 USA. E-mail: \\ mathfg@mizzou1.missouri.edu \\ ${ }^{2}$ Department of Mathematical Sciences, The Norwegian Institute of Technology, University of \\ Trondheim, N-7034 Trondheim, Norway. E-mail: holden@imf.unit.no \\ ${ }^{3}$ Division of Physics, Mathematics and Astronomy, California Institute of Technology, 253-37, \\ Pasadena, CA 91125 USA. This material is based upon work supported by the National Science \\ Foundation under Grant No. DMS-9101715. The Government has certain rights in this material
}

Received: 4 February 1994

Abstract: A recently established general trace formula for one-dimensional Schrödinger operators is systematically studied in the context of short-range potentials, potentials which approach different spatial asymptotes sufficiently fast, and appropriate impurity (defect) interactions in one-dimensional solids. We prove the absolute summability of the trace formula and establish its connections with scattering quantities, such as reflection coefficients, in each case.

\section{Introduction}

This paper is the third in a series on a general trace formula and its ramifications in (inverse) spectral theory for one-dimensional Schrödinger operators started in [16] and continued in [19]. The main theme in [16] concentrates around a general trace formula for self-adjoint Schrödinger operators $H$ in $L^{2}(\mathbb{R})$ of the type

$$
H=-\frac{d^{2}}{d x^{2}}+V
$$

where we assume that the real-valued potential $V$ is continuous and bounded from below. In order to gain some information on $V(y)$, we shall compare $H$ with the associated self-adjoint Dirichlet operator $H_{y}^{D}$ obtained from $H$ by imposing an additional Dirichlet boundary condition $\lim _{\varepsilon \downarrow} \psi \psi(y \pm \varepsilon)=0$ at the point $y \in \mathbb{R}$. Since the resolvent difference $\left[\left(H_{y}^{D}-z\right)^{-1}-(H-z)^{-1}\right]$ is rank one (cf. (2.37)), Krein's spectral shift function $\xi(\lambda, y)[25,33]$ for the pair $\left(H_{y}^{D}, H\right)$ exists for all $y \in \mathbb{R}$ and a.e. $\lambda \in \mathbb{R}$ (with respect to Lebesgue measure) and one obtains for all $y \in \mathbb{R}$,

$$
\begin{gathered}
\operatorname{Tr}\left[f\left(H_{y}^{D}\right)-f(H)\right]=\int_{\mathbb{R}} d \lambda f^{\prime}(\lambda) \xi(\lambda, y), \\
0 \leqq \xi(\lambda, y) \leqq 1 \quad \text { a.e. } \lambda \in \mathbb{R} \\
\xi(\lambda, v)=0 \quad \lambda<\inf \sigma(H)
\end{gathered}
$$


for any $f \in C^{2}(\mathbb{R})$ with $\left(1+\lambda^{2}\right) f^{(j)} \in L^{2}((0, \infty)), j=1,2$ and $f(\lambda)=(\lambda-z)^{-1}$, $z \in \mathbb{C} \backslash[\inf \sigma(H), \infty)$. (Here $\sigma(\cdot)$ denotes the spectrum.) A closer look at the rankone resolvent difference of $H_{y}^{D}$ and $H$ reveals the additional result that for each $y \in \mathbb{R}$ and a.e. $\lambda \in \mathbb{R}$,

$$
\xi(\lambda, y)=\lim _{\varepsilon \downarrow 0} \pi^{-1} \operatorname{Im}\{\ln [G(\lambda+i \varepsilon, y, y)]\},
$$

where $G\left(z, x, x^{\prime}\right)$ denotes the Green's function of $H$ (i.e., the integral kernel of $\left.(H-z)^{-1}\right)$. The main results proven in $[16,19]$ then revolve around the following general trace formula.

Theorem 1.1. [16, 19] Let $V$ be a measurable function on $\mathbb{R}$ satisfying

(i) $\sup _{n \in \mathbb{N}} \int_{n}^{n+1} d x\left|V_{-}(x)\right|<\infty$,

(ii) $\int_{n}^{n+1} d x\left|V_{+}(x)\right|<\infty$ for all $n \in \mathbb{N}$,

where $V_{ \pm}(x)=[|V(x)| \pm V(x)] / 2$ and suppose $E_{o} \leqq$ inf $\sigma(H)$. If $x$ is a point of Lebesgue continuity for $V$, then

$$
V(x)=E_{o}+\lim _{\varepsilon \downarrow 0} \int_{E_{o}}^{\infty} d \lambda e^{-\varepsilon \lambda}[1-2 \xi(\lambda, x)] .
$$

The proof of (1.6) combines (1.2) for $f(\lambda)=e^{-\varepsilon \lambda}, \varepsilon>0$ with path integral arguments to control the trace of the heat kernel difference as $\varepsilon \downarrow 0$.

In the particularly simple case $V(x) \equiv 0, G(\lambda, x, x)=i / \lambda^{1 / 2}, \operatorname{Im}\left(\lambda^{1 / 2}\right) \geqq 0$ for $\lambda \geqq 0$, and hence $\xi(\lambda, x)=\left\{\begin{array}{l}1 / 2, \lambda>0 \\ 0, \lambda<0\end{array}\right.$. Further explicit examples can be found in Remark 2.5 in the context of reflectionless ( $N$-soliton) potentials and in (4.18)(4.20) in connection with periodic potentials. In fact, historically, after the pioneering work by Gel'fand and Levitan [12] on regularized traces for Schrödinger operators on a compact interval, the trace formula (4.19) for periodic (and certain classes of almost periodic) potentials was one of the two previously systematically studied trace formulae of the type (1.6) for Schrödinger operators on the whole real line (see, e.g., $[8,11,22,30,34]$ and more recently $[5,24,27,28]$ ). The other case studied in detail by Deift and Trubowitz [7] in 1979 was concerned with shortrange potentials $V(x)$ decaying sufficently fast as $|x| \rightarrow \infty$ under the assumption that $H=-\frac{d^{2}}{d x^{2}}+V$ has no eigenvalues. They proved that

$$
V(x)=\frac{2 i}{\pi} \int_{-\infty}^{\infty} d k k \ln \left[1+R(k) \frac{f_{+}(k, x)}{f_{-}(k, x)}\right]
$$

(where $f_{ \pm}(k, x)$ are the Jost functions at energy $E=k^{2}$ and $R(k)$ is a reflection coefficient) which is an analog of (1.6). In the special case of positive $C^{\infty}$-potentials of compact support, a trace formula of the type

$$
V(x)=\int_{0}^{\infty} d \lambda[1-2 \xi(\lambda, x)], \quad x \in \mathbb{R}
$$

has recently been found by Venakides [35]. However, the equivalence of (1.7) and (1.8) was not established in [35]. Moreover, $\xi(\lambda, x)$ was not identified as Krein's 
spectral shift function for the pair $\left(H_{x}^{D}, H\right)$ and also the connection (1.5) between $\xi(\lambda, x)$ and the Green's function of $H$ was not made.

The analog of the trace formula (1.6) and the associated formalism for secondorder finite-difference (Jacobi) operators, a summability result for operators $H$ with purely discrete spectrum along with a powerful new characterization of the absolutely continuous spectrum $\sigma_{\mathrm{ac}}(H)$ of $H$ as

$$
\sigma_{\mathrm{ac}}(H)=\overline{\{\lambda \in \mathbb{R} \mid 0<\xi(\lambda, x)<1\}^{\text {ess }}}
$$

for each fixed $x \in \mathbb{R}$ and some of its applications to the almost Mathieu equation or Harper's model (here -ess denotes the essential closure) are presented in the first paper [16] of our series.

The case of general self-adjoint boundary conditions of the type $\psi^{\prime}(x)+\beta \psi(x)=$ $0, \beta \in \mathbb{R} \cup\{\infty\}$ together with trace formulas for all higher-order $\mathrm{KdV}$ invariants (expressed as differential polynomials in $V$ ) are studied in detail in the second paper [19] of our series.

In the present third paper of our series, we shall give a systematic study of short-range perturbations in which the regularization (Abelian limit) $\varepsilon \downarrow 0$ in (1.6) can be removed and prove the absolute summability of the trace formula (1.6). Specifically, we shall study the following three situations:

(i) Sufficiently short-range potentials with certain regularity properties (typically, $V \in H^{2,1}(\mathbb{R})$, see $\left.(2.1)\right)$ in Sect. 2.

(ii) Potentials which tend to different asymptotes as $x \rightarrow \pm \infty$ sufficiently fast in Sect. 3 .

(iii) Impurity (defect) scattering in connection with potentials of the type $V=$ $V^{o}+W$, where $V^{o}(x+a)=V^{o}(x)$ for some $a>0$ represents the periodic background and the short-range perturbation $W$ models impurities (defects) in a one-dimensional crystal, are treated in Sect. 4.

In each of these three situations, we establish the connection between $\xi(\lambda, x)$ and appropriate scattering quantities, such as reflection coefficients and Jost functions, and prove the absolute summability of the trace formula (1.6),

$$
\int_{R}^{\infty} d \lambda|1-2 \xi(\lambda, x)|<\infty, \quad R \in \mathbb{R}
$$

removing the Abelian limit $\varepsilon \downarrow 0$ in (1.6).

It should be pointed out at this occasion that the Abelian limit $\varepsilon \downarrow 0$ in (1.6) cannot be removed in general if $V(x) \rightarrow \infty$ as $x \rightarrow \infty$ or $x \rightarrow-\infty$ irrespective of the regularity properties of $V(x)$. This is particularly plain in the case where $V(x) \rightarrow \infty$ as $x \rightarrow \pm \infty$, since then for each $x \in \mathbb{R},|1-2 \xi(\lambda, x)|=1$ for a.e. $\lambda \in \mathbb{R}$. But even if $V(x)$ tends to a constant sufficiently fast as $x \rightarrow-\infty$ and $V(x) \rightarrow \infty$, explicit examples (such as, e.g., $V(x)=e^{x}$ ) in [26] show that $[1-2 \xi(., x)] \notin L^{1}((R, \infty) ; d \lambda), R, x \in \mathbb{R}$. In these situations, the Abelian limit $\varepsilon \downarrow 0$ in (1.6) represents a genuine summability method.

The fourth paper [17] in our series is devoted to various multidimensional trace formulas in terms of heat kernel asymptotics. A brief announcement of our results appeared in [18], expository accounts of this circle of ideas can be found in [14, 33]. Papers exploring several new solutions of inverse spectral problems are in preparation. 


\section{Short-Range Potentials}

In this section we illustrate the trace formula (1.6) in the particular case of shortrange potentials satisfying

$$
V \in H^{2,1}(\mathbb{R}), \quad V \text { real-valued } .
$$

Here $H^{m, p}(\mathbb{R}), m, p \in \mathbb{N}$ denotes the usual Sobolev space whose elements have up to $m$ distributional derivatives in $L^{p}(\mathbb{R})$. The regularity condition on $V$ in $(2.1)$ is essential in connection with our main result in Theorem 2.3, the removal of a regularization procedure (Abelian limit) in our trace formula (1.6) (cf. (2.39)(2.41)).

The associated self-adjoint Schrödinger operator $H$ in $L^{2}(\mathbb{R})$ is then defined by

$$
H=-\frac{d^{2}}{d x^{2}}+V, \quad \mathcal{D}(H)=H^{2,2}(\mathbb{R}),
$$

and the spectrum $\sigma(H)$ of $H$ is of the type

$$
\sigma(H)=\sigma_{d}(H) \cup[0, \infty), \quad \sigma_{\text {ess }}(H)=[0, \infty) .
$$

Here $\sigma_{\text {ess }}(H)$ is the essential spectrum of $H$ and the discrete spectrum $\sigma_{d}(H)$ of $H$ is a bounded subset of $(-\infty, 0)$ which may be empty, finite, or countably infinite. We denote the latter by

$$
\sigma_{d}(H)=\left\{e_{j}\right\}_{j \in J}, \quad e_{j}<e_{j+1},
$$

where

$$
J=\left\{\begin{array}{l}
\emptyset \\
\{0,1,2, \ldots, N\} \\
\mathbb{N}_{0}=\mathbb{N} \cup\{0\}
\end{array}\right.
$$

is an appropriate index set. For later purposes we will also need the notation

$$
J_{+}=\left\{\begin{array}{l}
\emptyset \\
\{1,2, \ldots, N, N+1\}, \quad e_{N+1}=0 \\
\mathbb{N}
\end{array}\right.
$$

depending on whether $J$ is empty, finite, or infinite. We also remark that each eigenvalue of $H$ is simple, $H$ has no eigenvalues embedded into $(0, \infty)$, and the spectrum of $H$ in $(0, \infty)$ is purely absolutely continuous and of uniform multiplicity two under hypothesis (2.1). It should perhaps be noted that the weak falloff condition of $V(x)$ as $|x| \rightarrow \infty$ in (2.1) admits situations where zero is a (necessarily simple) eigenvalue of $H$ though these cases can easily be excluded by adding the assumption $V \in L^{1}(\mathbb{R} ;(1+|x|) d x)$ in $(2.1)$. For details in connection with these spectral properties of $H$, see, for example, $[4,6,7,13]$, and the references therein.

In addition to $H$ we also need to introduce the closely associated self-adjoint Dirichlet operator $H_{y}^{D}$ in $L^{2}(\mathbb{R})$ defined by

$$
H_{y}^{D}=-\frac{d^{2}}{d x^{2}}+V, \mathcal{D}\left(H_{y}^{D}\right)=\left\{g \in H^{1,2}(\mathbb{R}) \cap H^{2,2}(\mathbb{R} \backslash\{y\}) \mid g(y)=0\right\}, \quad y \in \mathbb{R} .
$$


The spectrum of $H_{y}^{D}$ is then given by

$$
\sigma\left(H_{y}^{D}\right)=\sigma_{d}\left(H_{y}^{D}\right) \cup[0, \infty), \quad \sigma_{\mathrm{ess}}\left(H_{y}^{D}\right)=[0, \infty),
$$

where

$$
\begin{gathered}
\sigma_{d}\left(H_{y}^{D}\right)=\left\{\mu_{j}(y)\right\}_{j \in J_{+}} \cap\left(e_{0}, 0\right), \\
e_{0}<\mu_{1}(y) \leqq e_{1}, \quad e_{j-1} \leqq \mu_{j}(y) \leqq e_{j}, \quad j \in J_{+} \backslash\{1\} .
\end{gathered}
$$

(In the special case where $J_{+}=\{1,2, \ldots, N+1\}$ is finite and $\mu_{N+1}(y)=e_{N+1}=0$, our notation in (2.9) indicates that $\mu_{N+1}(y)=0$ is not a discrete Dirichlet eigenvalue though it may be a (non-isolated) eigenvalue of $H_{y}^{D}$ due to. our weak falloff conditions on $V$ as $|x| \rightarrow \infty$.) In particular,

$$
H_{y}^{D} \geqq H
$$

Since the resolvent of $H_{y}^{D}$ is a rank-one perturbation of that of $H$ (see (2.37)) and

$$
H_{y}^{D}=H_{-, y}^{D} \oplus H_{+, y}^{D},
$$

where $H_{ \pm, y}^{D}$ denote the corresponding half-line Dirichlet operators in $L^{2}((y, \pm \infty))$, the spectrum of $H_{y}^{D}$ in $(0, \infty)$ is purely absolutely continuous and of uniform multiplicity two. The discrete spectrum $\sigma_{d}\left(H_{y}^{D}\right)$ however, in contrast to that of $H$, is not necessarily simple. More precisely, $\mu_{j}(y)$ is a simple eigenvalue of $H_{y}^{D}$ if and only if $e_{j-1}<\mu_{j}(y)<e_{j}$. In this case, $\mu_{j}(y)$ is a (simple) eigenvalue of either $H_{-, y}^{D}$ or $H_{+, y}^{D}$, but not of both. Whenever $\mu_{j}(y) \in\left\{e_{j-1}, e_{j}\right\}$ (possibly excluding the case $\mu_{N+1}(y)=0$ as explained after (2.9)), the multiplicity of $\mu_{j}(y)$ is two and $\mu_{j}(y)$ is a (simple) eigenvalue of both $H_{-, y}^{D}$ and $H_{+, y}^{D}$.

As a final preparation for the main results of this section, we briefly recall a few basic formulas in connection with scattering theory for the pair $\left(H, H_{o}\right)$, where $H_{o}=-\frac{d^{2}}{d x^{2}}, \mathcal{D}\left(H_{o}\right)=H^{2,2}(\mathbb{R})$. Details can be found, for example, in [2-4, 7, 13, 28], Ch. 6, [29], Sect. 3.5, and the references therein. The Jost solutions $f_{ \pm}(z, x)$ of $H$ are defined by

$$
\begin{gathered}
f_{ \pm}(z, x)=e^{ \pm t z^{1 / 2} x}-\int_{x}^{ \pm \infty} d x^{\prime} z^{-1 / 2} \sin \left[z^{1 / 2}\left(x-x^{\prime}\right)\right] V\left(x^{\prime}\right) f_{ \pm}\left(z, x^{\prime}\right), \\
z \in \mathbb{C} \backslash\{0\}, \operatorname{Im}\left(z^{1 / 2}\right) \geqq 0, x \in \mathbb{R},
\end{gathered}
$$

such that

$$
H f_{ \pm}(z, x)=z f_{ \pm}(z, x), \quad z \in \mathbb{C} \backslash\{0\}
$$

in the distributional sense. The unitary scattering matrix $S(\lambda), \lambda>0$ in $\mathbb{C}^{2}$ associated with the pair $\left(H, H_{o}\right)$ then explicitly reads in terms of transmission and reflection coefficients from left and right incidence

$$
S(\lambda)=\left(\begin{array}{cc}
T(\lambda) & R^{r}(\lambda) \\
R^{l}(\lambda) & T(\lambda)
\end{array}\right), \quad \lambda>0,
$$


where

$$
\begin{aligned}
& T(\lambda)=\frac{2 i \lambda^{1 / 2}}{W\left(f_{-}(\lambda), f_{+}(\lambda)\right)}=\left[1-\frac{1}{2 i \lambda^{1 / 2}} \int_{\mathbb{R}} d x V(x) e^{ \pm i \lambda^{1 / 2} x} f_{\mp}(\lambda, x)\right]^{-1}, \\
& R^{l}(\lambda)=-\frac{W\left(\overline{f_{-}(\lambda),}, f_{+}(\lambda)\right)}{W\left(f_{-}(\lambda), f_{+}(\lambda)\right)}=\frac{T(\lambda)}{2 i \lambda^{1 / 2}} \int_{\mathbb{R}} d x V(x) e^{i \lambda^{1 / 2} x} f_{+}(\lambda, x), \\
& R^{r}(\lambda)=-\frac{W\left(f_{-}(\lambda), \overline{\left.f_{+}(\lambda)\right)}\right.}{W\left(f_{-}(\lambda), f_{+}(\lambda)\right)}=\frac{T(\lambda)}{2 i \lambda^{1 / 2}} \int_{\mathbb{R}} d x V(x) e^{-i \lambda^{1 / 2} x} f_{-}(\lambda, x),
\end{aligned}
$$

where

$$
f_{ \pm}(\lambda, x)=\lim _{\varepsilon \downarrow 0} f_{ \pm}(\lambda+i \varepsilon, x), \quad \lambda>0
$$

and $W(f, g)(x)=f(x) g^{\prime}(x)-f^{\prime}(x) g(x)$ denotes the Wronskian of $f$ and $g$. In addition, we recall that

$$
T(\lambda) f_{ \pm}(\lambda, x)=R^{r}(\lambda) f_{\mp}(\lambda, x)+\overline{f_{\mp}(\lambda, x)}, \quad \lambda>0
$$

and that the Green's function of $H$ (the integral kernel of $\left.(H-z)^{-1}\right)$ is given by

$$
G\left(z, x, x^{\prime}\right)=\frac{f_{+}(z, x) f_{-}\left(z, x^{\prime}\right)}{W\left(f_{+}(z), f_{-}(z)\right)}, \quad x^{\prime} \leqq x, z \in \mathbb{C} \backslash\{0\}, \operatorname{Im}\left(z^{1 / 2}\right) \geqq 0 .
$$

Specializing to $x=x^{\prime}, G(z, x, x)$ is well known to be Herglotz function in $z \in \mathbb{C}_{+}=$ $\{z \in \mathbb{C} \mid \operatorname{Im}(z)>0\}$ for all $x \in \mathbb{R}$, that is, $G(z, x, x)$ is analytic in $\mathbb{C}_{+}$and

$$
\operatorname{Im}[G(z, x, x)]>0, \quad \overline{G(z, x, x)}=G(\bar{z}, x, x), \quad z \in \mathbb{C}_{+}, x \in \mathbb{R} .
$$

As a consequence (see, e.g., [1] or, for a different approach, [16, 33]) $G(z, x, x)$ admits the exponential representation.

$$
G(z, x, x)=\exp \left[c+\int_{\mathbb{R}}\left[\frac{1}{\lambda-z}-\frac{\lambda}{1+\lambda^{2}}\right] \xi(\lambda, x) d \lambda\right], \quad z \in \mathbb{C}_{+}, x \in \mathbb{R}
$$

where

$$
c \in \mathbb{R}, \quad 0 \leqq \xi(\lambda, x) \leqq 1 \text { for a.e. } \lambda \in \mathbb{R}
$$

Fatou's lemma then implies that

$$
\xi(\lambda, x)=\lim _{\varepsilon \downarrow o} \pi^{-1} \operatorname{Im}\{\ln [G(\lambda+i \varepsilon, x, x)]\}
$$

exists for all $x \in \mathbb{R}$ and a.e. $\lambda \in \mathbb{R}$. The normalization

$$
\xi(\lambda, x)=0, \quad \lambda<E_{o}=\inf \sigma(H)
$$

is then consistent with (2.23), (2.10) and the fact that

$$
G(\lambda+i 0, x, x)>0, \quad \lambda<E_{o} .
$$


As pointed out in Theorem 1.1 of the introduction, the general trace formula for $V \in C(\mathbb{R}), V$ bounded from below, proven in [16] then reads

$$
V(x)=E_{o}+\lim _{\varepsilon \downarrow 0} \int_{E_{o}}^{\infty} d \lambda e^{-\varepsilon \lambda}[1-2 \xi(\lambda, x)], \quad x \in \mathbb{R} .
$$

Before we discuss how to remove the Abelian limit in (2.27) in the present shortrange case, it should be pointed out that $\xi(\lambda, x)$ is Krein's spectral shift function [25] for the pair $\left(H_{x}^{D}, H\right)$. In particular, for $\lambda \in \sigma_{\mathrm{ac}}(H)^{o}\left(A^{o}\right.$ denotes the interior of a subset $A \subset \mathbb{R}), \xi(\lambda, x)$ is essentially the scattering phase shift for the pair $\left(H_{x}^{D}, H\right)$ since one verifies that

$$
\operatorname{det}\left[S\left(\lambda, H_{x}^{D}, H\right)\right]=\frac{\overline{G(\lambda+i 0, x, x)}}{G(\lambda+i 0, x, x)}=e^{-2 \pi i \xi(\lambda, x)}, \quad \lambda \in \sigma_{\mathrm{ac}}(H)^{o},
$$

where $S\left(\lambda, H_{x}^{D}, H\right)$ denotes the unitary scattering matrix in $\mathbb{C}^{2}$ for the pair $\left(H_{x}^{D}, H\right)$.

We start our analysis with the following lemma.

Lemma 2.1. Suppose $V \in H^{2,1}(\mathbb{R})$ is real-valued. Then for all $x \in \mathbb{R}$,

$$
\begin{gathered}
\xi(\lambda, x)=0, \quad \lambda<E_{o}=\inf \sigma(H), \\
\xi(\lambda, x)=\frac{1}{2}+\pi^{-1} \operatorname{Im}\left\{\ln \left[1+R^{\stackrel{r}{l}}(\lambda) \frac{f_{ \pm}(\lambda, x)^{2}}{\left|f_{ \pm}(\lambda, x)\right|^{2}}\right]\right\}, \quad \lambda>0 .
\end{gathered}
$$

In particular, $\xi(\lambda, x)$ is continuous for $\lambda>0$. Moreover,

$$
|1-2 \xi(\lambda, x)| \leqq\left|R^{r(l)}(\lambda)\right|, \quad \lambda>0
$$

and

$$
[1-2 \xi(\lambda, x)] \underset{\lambda \rightarrow \infty}{=} o\left(\lambda^{-3 / 2}\right)
$$

uniformly with respect to $x \in \mathbb{R}$. In addition,

$$
\begin{gathered}
\xi(\lambda, x)=\left\{\begin{array}{ll}
0, & \text { if } \lambda<e_{0} \text { or if } \mu_{j}(x)<\lambda<e_{j} \\
1, & \text { if } e_{j-1}<\lambda
\end{array} \text { if } \mu_{j}(x) \in\left(e_{j-1}, e_{j}\right),\right. \\
\xi(\lambda, x)= \begin{cases}0, & \text { if } e_{j-1}<\lambda<e_{j} \text { and } \mu_{j}(x)=e_{j} \\
1, & \text { if } e_{j-1}<\lambda<e_{j} \text { and } \mu_{j}(x)=e_{j-1}\end{cases}
\end{gathered}
$$

whenever $\sigma_{d}(H) \neq \emptyset$.

Proof. Equation (2.30) follows from (2.24) and

$$
\begin{aligned}
G(\lambda+i 0, x, x) & =-\frac{T(\lambda)}{2 i \lambda^{1 / 2}} f_{+}(\lambda, x) f_{-}(\lambda, x) \\
& =\left(i / 2 \lambda^{1 / 2}\right)\left|f_{ \pm}(\lambda, x)\right|^{2}\left[1+R^{l}(\lambda) \frac{f_{ \pm}(\lambda, x)^{2}}{\left|f_{ \pm}(\lambda, x)\right|^{2}}\right], \quad \lambda>0,
\end{aligned}
$$

which in turn is implied by (2.15), (2.18)-(2.20). Continuity of $\xi(\lambda, x)$ for $\lambda>0$ follows from the fact that $G(\lambda+i 0, x, x)$ is continuous and zero-free for $\lambda \in(0, \infty)$. Inequality (2.31) follows from (2.30) and an elementary geometrical argument. 
(In fact, $\left|\arg \left(1+R e^{i \varphi}\right)\right| \leqq \arcsin (|R|) \leqq \pi / 2$ for $|R| \leqq 1, \varphi \in \mathbb{R}$ and $\sin (x) \geqq 2 x / \pi$ for $0 \leqq x \leqq \pi / 2$ imply $\left|\arg \left(1+R e^{l \varphi}\right)\right| \leqq \pi|R| / 2$.) Relation (2.32) is then implied by $(2.31)$ and

$$
R^{r(l)}(\lambda) \underset{\lambda \rightarrow \infty}{=} o\left(\lambda^{-3 / 2}\right),
$$

which is a consequence of (2.15)-(2.17) and two integrations by parts applying the Riemann-Lebesgue lemma. Relation (2.33) directly follows from (2.24) and the fact that $G(z, x, x)$ is real-valued for $z<0$ with zeros precisely at the Dirichlet eigenvalues $\mu_{j}(x)$ of $H_{x}^{D}$ since

$$
\begin{aligned}
\left(H_{x}^{D}-z\right)^{-1}= & \left.(H-z)^{-1}-G(z, x, x)^{-1}(\overline{G(z, x, .}), .\right) G(z, ., x), \\
& z \in \mathbb{C} \backslash\left\{\sigma\left(H_{x}^{D}\right) \cup \sigma(H)\right\} .
\end{aligned}
$$

Here $(\cdot, \cdot)$ denotes the scalar product in $L^{2}(\mathbb{R})$. Relation (2.34) then follows from (2.33) by a continuity argument.

Remark 2.2. (i) Inequality (2.31) holds for any real-valued potential satisfying $V \in L^{1}(\mathbb{R})$, and hence

$$
[1-2 \xi(\cdot, x)] \in L^{1}((0, \infty) ; d \lambda) \text { if } R^{r(l)} \in L^{1}((0, \infty) ; d \lambda) .
$$

In particular, $R^{r(l)} \in L^{1}((0, \infty) ; d \lambda)$ together with $(2.31)$ are all that's needed to remove the Abelian limit in (2.27) (see Theorem 2.3). We also note that (2.36) (and hence (2.32)) holds if $V^{\prime}$ is merely piecewise absolutely continuous admitting finitely-many jump discontinuities (i.e., there exists a finite partition of $\mathbb{R},-\infty=$ $x_{0}<x_{1}<\cdots<x_{M}<x_{M+1}=\infty$ such that $V^{\prime}$ is (locally) absolutely continuous on each interval $\left.\left(x_{m}, x_{m+1}\right), 0 \leqq m \leqq M\right)$.

(ii) Obviously, (2.33) and (2.34), in contrast to (2.30), are generally valid in spectral gaps of $H$ and by no means linked to the short-range nature of $V$ subject to (2.1). In particular, (2.33) is a general property of Krein's spectral function as long as $e_{j}$ and $\mu_{j}(x)$ are simple eigenvalues of $H$ and $H_{x}^{D}$, respectively.

Given Lemma 2.1, we can now remove the Abelian limit $\varepsilon \downarrow 0$ in the trace formula (2.27) for $V(x)$ and state the principal result of this section. (We recall our notational conventions in (2.4)-(2.6), (2.8), (2.9), and the paragraph following (2.9).)

Theorem 2.3. Suppose $V \in H^{2,1}(\mathbb{R})$ is real-valued and denote $E_{o}=\inf \sigma(H)$. Then $[1-2 \xi(., x)] \in L^{1}\left(\left(E_{o}, \infty\right) ; d \lambda\right), x \in \mathbb{R}$ and

$$
\begin{aligned}
V(x)= & E_{o}+\int_{E_{o}}^{\infty} d \lambda[1-2 \xi(\lambda, x)] \\
= & 2\left\{e_{o}+\sum_{j \in J_{+}}\left[e_{j}-\mu_{j}(x)\right]\right\}+\int_{0}^{\infty} d \lambda[1-2 \xi(\lambda, x)] \\
= & 2\left\{e_{0}+\sum_{j \in J_{+}}\left[e_{j}-\mu_{j}(x)\right]\right\} \\
& -(2 / \pi) \int_{0}^{\infty} d \lambda \operatorname{Im}\left\{\ln \left[1+R^{l}(\lambda) \frac{f_{ \pm}(\lambda, x)^{2}}{\left|f_{ \pm}(\lambda, x)\right|^{2}}\right]\right\}, x \in \mathbb{R} .
\end{aligned}
$$

If $\sigma_{d}(H)=\emptyset$, the discrete spectrum part $2\{\ldots\}$ in $(2.40)$ and $(2.41)$ is to be deleted. 
Proof. The trace formula (2.39) follows from (2.27), (2.31), and (2.32) applying the Lebesgue dominated convergence theorem. Equalities (2.40) and (2.41) are then obvious from (2.39), (2.30), (2.33), and (2.34).

Remark 2.4. Formula (2.41), in the special case $\sigma_{d}(H)=\emptyset$, is due to Deift and Trubowitz [7]. Formula (2.39), on the other hand, again in the special case $\sigma_{p}(H)=$ $\emptyset$ (more precisely for $0 \leqq V \in C_{0}^{\infty}(\mathbb{R})$ ), appeared in a paper by Venakides [35]. However, the connection (2.24) between $\xi(\lambda, x)$ and the Green's function $G(z, x, x)$ of $H$, and hence the connection between (2.39) and the earlier result (2.41) in [7], was not establised in [35]. Moreover, $\xi(\lambda, x)$ was not identified as Krein's spectral shift function for the pair $\left(H_{x}^{D}, H\right)$ in [35].

Remark 2.5. It seems worthwhile to point out the particularly simple step functionlike structure of $\xi(\lambda, x)$ in connection with reflectionless potentials characterized by $R^{r(l)}(\lambda) \equiv 0, \lambda>0$. In this case $\xi(\lambda, x)$ is given by (2.33), (2.34) for $\lambda<0$ and by

$$
\xi(\lambda, x)=\frac{1}{2}, \quad \lambda>0
$$

on the (interior of the) absolutely continuous spectrum of $H$. This applies, in particular, to all $N$-soliton potentials (including $V \equiv 0$ ) and to a class of $\infty$-soliton potentials (having infinitely-many negative eigenvalues accumulating at zero) introduced in $[20,21]$.

We conclude this section with a few remarks on the low-energy behavior of $\xi(\lambda, x)$ as $\lambda \downarrow 0$. Assuming, in addition to (2.1), that $V$ satisfies

$$
V \in L^{1}\left(\mathbb{R} ;\left(1+x^{2}\right) d x\right),
$$

we need to consider the following case distinctions:

Case I. $W\left(f_{-}(0), f_{+}(0)\right) \neq 0$ and $f_{-}(0, x) f_{+}(0, x) \neq 0$.

(The first requirement can be expressed as $\int_{\mathbb{R}} d x V(x) f_{ \pm}(0, x) \neq 0$ and is equivalent to the fact that $H$ has no threshold resonance; see, e.g. [2, 3]. The second requirement says $\left.\lim _{x^{\prime} \rightarrow x} \mu_{N+1}\left(x^{\prime}\right) \neq 0\right)$. Then

$$
\begin{gathered}
R^{r(l)}(\lambda) \underset{\lambda \downarrow 0}{=}-1+O\left(\lambda^{1 / 2}\right), \\
T(\lambda) \underset{\lambda \downarrow 0}{=} \frac{-2 i \lambda^{1 / 2}}{\int_{\mathbb{R}} d x^{\prime} V\left(x^{\prime}\right) f_{\mp}\left(0, x^{\prime}\right)}\left[1+O\left(\lambda^{1 / 2}\right)\right], \\
G(\lambda+i 0, x, x) \underset{\lambda \downarrow 0}{=} \frac{-f_{+}(0, x) f_{-}(0, x)}{\int_{\mathbb{R}} d x^{\prime} V\left(x^{\prime}\right) f_{ \pm}\left(0, x^{\prime}\right)}+O\left(\lambda^{1 / 2}\right),
\end{gathered}
$$

and hence

$$
\xi(\lambda, x)=\pi^{-1} \arg [G(\lambda+i 0, x, x)] \underset{\lambda \downarrow 0}{=} O\left(\lambda^{1 / 2}\right) \text { in case } \mathrm{I}
$$

since $f_{ \pm}(0, x)$ are real-valued.

Case II. $W\left(f_{-}(0), f_{+}(0)\right)=0$ and $f_{-}(0, x) f_{+}(0, x) \neq 0$. 
(The first requirement can be written as $\int_{\mathbb{R}} d x V(x) f_{ \pm}(0, x)=0$ and is equivalent to the fact that $H$ has a threshold resonance, see, e.g., [2, 3].) Then [2, 3]

$$
\begin{gathered}
R^{l}(\lambda) \underset{\lambda \downarrow 0}{=} \mp \frac{2 c_{1} \overline{c_{2}}}{\left|c_{1}\right|^{2}+\left|c_{2}\right|^{2}}+O\left(\lambda^{1 / 2}\right), \\
T(\lambda) \underset{\lambda \downarrow 0}{=} \frac{\left|c_{1}\right|^{2}-\left|c_{2}\right|^{2}}{\left|c_{1}\right|^{2}+\left|c_{2}\right|^{2}}+O\left(\lambda^{1 / 2}\right),
\end{gathered}
$$

with $\left|c_{1}\right| \neq\left|c_{2}\right|$ and hence $R^{r(l)}(0) \neq-1$. Thus

$$
G(\lambda+i 0, x, x) \underset{\lambda \downarrow 0}{=}\left(i / 2 \lambda^{1 / 2}\right)\left|f_{ \pm}(0, x)\right|^{2}\left[1+R^{l}(0)\right]+O(1)
$$

and hence

$$
\xi(\lambda, x)=\pi^{-1} \arg [G(\lambda+i 0, x, x)] \underset{\lambda \downarrow 0}{=} \frac{1}{2}+O\left(\lambda^{1 / 2}\right) \text { in case II } .
$$

We emphasize that $V \equiv 0$ and more generally, all $N$-soliton potentials $V_{N}$ mentioned in Remark 2.5 have a zero-energy resonance and hence belong to case II.

The case where $f_{-}(0, x) f_{+}(0, x)=0$ can be dealt with analogously, but requires higher-order computations.

\section{Cascades}

As the title of this section suggests, we shall now indicate how to extend the results of Sect. 2 to potentials with non-trivial spatial asymptotics. More precisely, we shall assume that $V$ satisfies

$$
\begin{array}{r}
V, V^{\prime} \in A C_{\mathrm{loc}}(\mathbb{R}), \quad V \text { real-valued, } V^{\prime}, V^{\prime \prime} \in L^{1}(\mathbb{R}), \\
\int_{-\infty}^{0} d x|V(x)|+\int_{0}^{\infty} d x\left|V(x)-V_{+}\right|<\infty \text { for some } V_{+}>0
\end{array}
$$

throughout the major part of this section. (By reflection, $x \rightarrow-x$, it suffices to consider $V_{+}>0$.)

Since most of the details will be similar to those in the previous section, we shall mostly refer to Sect. 2 for notations and basic facts and dwell only on situations markedly different in the present context of (3.1).

Introducing $H, H_{y}^{D}, J, J_{+}$, etc. as in Sect. 2, the absolutely continuous spectrum of $H$ and $H_{y}^{D}$ now equals $[0, \infty)$ with uniform spectral multiplicity one on $\left(0, V_{+}\right)$ and two on $\left(V_{+}, \infty\right)$. While $H$ has no embedded eigenvalues in $(0, \infty), H_{y}^{D}$ may have (countably infinitely-many) eigenvalues in $\left[0, V_{+}\right]$as briefly discussed in Remark 3.4.

Concerning the stationary scattering theory for $H$, one has to replace the Jost solutions (2.12) by

$$
\begin{gathered}
f_{ \pm}(z, x)=e^{ \pm i k_{ \pm} x}-\int_{x}^{ \pm \infty} d x^{\prime} k_{ \pm}^{-1} \sin \left[k_{ \pm}\left(x-x^{\prime}\right)\right]\left[V\left(x^{\prime}\right)-V_{ \pm}\right] f_{ \pm}\left(z, x^{\prime}\right), \\
k_{+}(z)=\left(z-V_{+}\right)^{1 / 2}, k_{-}(z)=z^{1 / 2}, V_{-}=0, \operatorname{Im}\left[k_{ \pm}(z)\right] \geqq 0, z \in \mathbb{C} \backslash\left\{0, V_{+}\right\}, x \in \mathbb{R}
\end{gathered}
$$


to obtain

$$
H f_{ \pm}(z, x)=z f_{ \pm}(z, x), \quad z \in \mathbb{C} \backslash\left\{0, V_{+}\right\}
$$

in the distributional sense. The unitary scattering matrix $S(\lambda), \lambda>0$ in $\mathbb{C}$, resp. $\mathbb{C}^{2}$, now reads as follows:

$$
S(\lambda)=R^{l}(\lambda)=-\frac{\overline{W\left(f_{-}(\lambda), f_{+}(\lambda)\right)}}{W\left(f_{-}(\lambda), f_{+}(\lambda)\right)}, \quad 0<\lambda<V_{+}
$$

(note that $S(\lambda)$ is unimodular in this case since $f_{+}(\lambda, x)$ is real-valued for $0<\lambda<$ $\left.V_{+}\right)$and

$$
S(\lambda)=\left(\begin{array}{cc}
T(\lambda) & R^{r}(\lambda) \\
R^{l}(\lambda) & T(\lambda)
\end{array}\right), \quad \lambda>V_{+}
$$

where

$$
\begin{aligned}
T(\lambda) & =\frac{2 i\left[k_{+}(\lambda) k_{-}(\lambda)\right]^{1 / 2}}{W\left(f_{-}(\lambda), f_{+}(\lambda)\right)}, \\
R^{l}(\lambda) & =-\frac{W\left(\overline{f_{-}(\lambda)}, f_{+}(\lambda)\right)}{W\left(f_{-}(\lambda), f_{+}(\lambda)\right)}, \\
R^{r}(\lambda) & =-\frac{W\left(f_{-}(\lambda), \overline{f_{+}(\lambda)}\right)}{W\left(f_{-}(\lambda), f_{+}(\lambda)\right)}
\end{aligned}
$$

Here $f_{ \pm}(\lambda, x)=\lim _{\varepsilon \downarrow 0} f_{ \pm}(\lambda+i \varepsilon, x)$ and the Green's function $G(z, x, x)$ of $H$ now satisfies

$$
\begin{aligned}
G(\lambda+i 0, x, x)= & \frac{f_{+}(\lambda, x) f_{-}(\lambda, x)}{W\left(f_{+}(\lambda), f_{-}(\lambda)\right)} \\
= & {\left[i / 2 k_{ \pm}(\lambda)\right]\left|f_{ \pm}(\lambda, x)\right|^{2}\left[1+R^{l}(\lambda) \frac{f_{ \pm}(\lambda, x)^{2}}{\left|f_{ \pm}(\lambda, x)\right|^{2}}\right], } \\
& \lambda>\left\{\begin{array}{ll}
V_{+}, & x \in \mathbb{R} . \\
0
\end{array},\right.
\end{aligned}
$$

The above expression for $G(\lambda+i 0, x, x)$ involving $R^{r}(\lambda)$ (as opposed to that involving $R^{l}(\lambda)$ ) appears to be singular at $\lambda=V_{+}$, since $k_{+}(\lambda)^{-1}=\left(\lambda-V_{+}\right)^{-1 / 2}$ (whereas $\left.k_{-}\left(V_{+}\right)^{-1}=V_{+}^{-1 / 2}\right)$. However, this apparent contradiction is easily resolved by observing that $R^{r}(\lambda) \underset{\lambda \downarrow V_{+}}{=}-1+o(1)$ (see also (3.33) for more details).

Remark 3.1. Scattering theory for potentials with different spatial asymptotics has been studied in detail, for example, in $[4,6,13]$, and we have freely used these results in (3.2)-(3.9). That $S(\lambda)$ for $0<\lambda<V_{+}$is unimodular in (3.4) illustrates the fact that total reflection occurs from left incidence in this energy regime as explored in detail in [6] (see also [13]).

Since relations (2.20)-(2.27) are independent of the short-range nature of $V$, they apply in the present case. In particular, the definition of $\xi(\lambda, x)$ in $(2.24)$ and the trace formula (2.27) remain valid. Similarly, Lemma 2.1 extends to potentials subject to the hypothesis (3.1) with only one minor change. 
Lemma 3.2. Suppose V satisfies the conditions (3.1). Then (2.29), (2.31)-(2.34) are valid in the present case. Equation (2.30) turns into

$$
\xi(\lambda, x)=\frac{1}{2}+\pi^{-1} \operatorname{Im}\left\{\ln \left[1+R^{r}(\lambda) \frac{f_{ \pm}(\lambda, x)^{2}}{\left|f_{ \pm}(\lambda, x)\right|^{2}}\right]\right\}, \quad \lambda>\left\{\begin{array}{l}
V_{+} \\
0
\end{array},\right.
$$

and $\xi(\lambda, x)$ is continuous in $\lambda>V_{+}$.

Proof. Except for the analog of (2.32), which follows again from

$$
R^{r(l)}(\lambda) \underset{\lambda \rightarrow \infty}{=} o\left(\lambda^{-3 / 2}\right)
$$

everything else is proven as in Lemma 2.1. The actual proof of (3.11), however, is now more cumbersome since no simple formulas such as the right-hand sides in (2.15)-(2.17) appear to be available in the present case. Hence, we briefly sketch a different (though straightforward) approach to (3.11) (following Lemma 2.3 in [13]). From the outset it is readily verified that

$$
\begin{aligned}
& T(\lambda)=1+O\left(\lambda^{-1 / 2}\right), \\
& R^{r(l)}(\lambda) \underset{\lambda \rightarrow \infty}{=} O\left(\lambda^{-1 / 2}\right) .
\end{aligned}
$$

In order to improve on (3.13), using the additional smoothness conditions on $V$, we explicitly compute the Wronskian of $\overline{f_{-}(\lambda, x)}$ and $f_{+}(\lambda, x)$ (for simplicity, at $x=0)$.

$$
\begin{aligned}
& W\left(\overline{f_{-}(\lambda)}, f_{+}(\lambda)\right)(0)=i\left(k_{+}-k_{-}\right)-\int_{0}^{\infty} d x \cos \left(k_{+} x\right)\left[V(x)-V_{+}\right] f_{+}(\lambda, x) \\
& -\int_{-\infty}^{0} d x \cos \left(k_{-} x\right) V(x) \overline{f_{-}(\lambda, x)}-\left(i k_{+} / k_{-}\right) \int_{-\infty}^{0} d x \sin \left(k_{-} x\right) V(x) \overline{f_{-}(\lambda, x)} \\
& -\left(i k_{-} / k_{+}\right) \int_{0}^{\infty} d x \sin \left(k_{+} x\right)\left[V(x)-V_{+}\right] f_{+}(\lambda, x) \\
& +k_{-}^{-1} \int_{-\infty}^{0} d x \sin \left(k_{-} x\right) V(x) \overline{f_{-}(\lambda, x)} \int_{0}^{\infty} d x^{\prime} \cos \left(k_{+} x^{\prime}\right)\left[V\left(x^{\prime}\right)-V_{+}\right] f_{+}\left(\lambda, x^{\prime}\right) \\
& -k_{+}^{-1} \int_{-\infty}^{0} d x \cos \left(k_{-} x\right) V(x) \overline{f_{-}(\lambda, x)} \int_{0}^{\infty} d x^{\prime} \sin \left(k_{+} x^{\prime}\right)\left[V\left(x^{\prime}\right)-V_{+}\right] f_{+}\left(\lambda, x^{\prime}\right), \lambda>V_{+} .
\end{aligned}
$$

Next, one observes

$$
\begin{gathered}
i\left[k_{+}(\lambda)-k_{-}(\lambda)\right] \underset{\lambda \rightarrow \infty}{=}\left(-i / 2 \lambda^{1 / 2}\right) V_{+}+O\left(\lambda^{-3 / 2}\right), \\
{\left[i k_{ \pm}(\lambda) / k_{\mp}(\lambda)\right] \underset{\lambda \rightarrow \infty}{=} i \mp(i / 2 \lambda) V_{+}+O\left(\lambda^{-2}\right),} \\
k_{ \pm}(\lambda)^{-1} \underset{\lambda \rightarrow \infty}{=} \lambda^{-1 / 2}+O\left(\lambda^{-3 / 2}\right),
\end{gathered}
$$


and

$$
\left|g_{ \pm}(\lambda, x)\right|+\left|g_{ \pm}^{\prime}(\lambda, x)\right|+\left|g_{ \pm}^{\prime \prime}(\lambda, x)\right| \leqq C, \quad x \leqq 0, \lambda \geqq V_{+}+1
$$

where

$$
g_{ \pm}(\lambda, x)=e^{\mp i k_{ \pm}(\lambda) x} f_{ \pm}(\lambda, x) .
$$

(The estimate (3.18) immediately follows from (3.2).) Employing (3.15)-(3.19), one arrives at

$$
W\left(\overline{f_{-}(\lambda)}, f_{+}(\lambda)\right) \underset{\lambda \rightarrow \infty}{=} o\left(\lambda^{-1}\right)
$$

after two integrations by parts in (3.14) (and a few tears) using the RiemannLebesgue lemma and

$$
\begin{aligned}
& g_{ \pm}(\lambda, 0) \underset{\lambda \rightarrow \infty}{=} 1 \mp\left(1 / 2 i \lambda^{1 / 2}\right) \int_{0}^{ \pm \infty} d x e^{\mp l k_{ \pm} x}\left[V(x)-V_{ \pm}\right] f_{ \pm}(\lambda, x)+O\left(\lambda^{-1}\right) \\
& g_{ \pm}^{\prime}(\lambda, 0) \underset{\lambda \rightarrow \infty}{=} O\left(\lambda^{-1 / 2}\right) .
\end{aligned}
$$

Combining (3.6)-(3.8), (3.12), and (3.20) then proves (3.11).

The asymptotic behavior (3.11) slightly improves Lemma 1.4 (iv) in [4] since we arrive at the conclusion $o\left(\lambda^{-3 / 2}\right)$ instead of $O\left(\lambda^{-3 / 2}\right)$ and their extra hypothesis $\int_{-\infty}^{0} d x(1+|x|)|V(x)|+\int_{0}^{\infty} d x(1+|x|)\left|V(x)-V_{+}\right|<\infty$ is not needed in our proof.

Remark 2.2 and the paragraph following it clearly apply in the present context.

Lemma 3.2 enables one to again remove the Abelian limit in the trace formula (1.6) for $V(x)$. (We recall our notational conventions in (2.4)-(2.6), (2.8), (2.9), and the paragraph following (2.9).)

Theorem 3.3. Suppose $V, V^{\prime} \in A C_{\mathrm{loc}}(\mathbb{R}), V$ is real-valued, $V^{\prime}, V^{\prime \prime} \in L^{1}(\mathbb{R})$, $\int_{-\infty}^{0} d x|V(x)|+\int_{0}^{\infty} d x\left|V(x)-V_{+}\right|<\infty$ for some $V_{+}>0$. Let $E_{o}=\inf \sigma(H)$. Then $[1-2 \xi(., x)] \in L^{1}\left(\left(E_{o}, \infty\right) ; d \lambda\right), x \in \mathbb{R}$ and

$$
\begin{aligned}
V(x)= & E_{o}+\int_{E_{o}}^{\infty} d \lambda[1-2 \xi(\lambda, x)] \\
= & 2\left\{e_{0}+\sum_{j \in J_{+}}\left[e_{J}-\mu_{j}(x)\right]\right\}+\int_{0}^{\infty} d \lambda[1-2 \xi(\lambda, x)] \\
= & 2\left\{e_{0}+\sum_{j \in J_{+}}\left[e_{j}-\mu_{j}(x)\right]\right\} \\
& -(2 / \pi) \int_{0}^{\infty} d \lambda \operatorname{Im}\left\{\ln \left[1+R^{l}(\lambda) \frac{f_{-}(\lambda, x)^{2}}{\left|f_{-}(\lambda, x)\right|^{2}}\right]\right\}, \quad x \in \mathbb{R} .
\end{aligned}
$$

If $\sigma_{p}(H)=\emptyset$, the discrete spectrum part $2\{\ldots\}$ in (3.24) and (3.25) is to be deleted.

Given Lemma 3.2, the proof of Theorem 3.3 is identical to that of Theorem 2.3. Remark 3.4. While $\xi(\lambda, x)$ is continuous in $\lambda>V_{+}, \xi(\lambda, x)$ may have (countably infinitely-many) jump discontinuities of size one in $\left[0, V_{+}\right]$. These discontinuities 
occur at those special energies $\mu_{J}(x) \in\left[0, V_{+}\right]$which are eigenvalues of the Dirichlet operator $H_{+, x}^{D}$ (the restriction of $H_{x}^{D}$ to $\left.(x, \infty)\right)$. The Green's function $G(z, x, x)$ of $H$ is of the type

$$
G(z, x, x)=\left[m_{x}^{-}(z)-m_{x}^{+}(z)\right]^{-1},
$$

where $m_{x}^{ \pm}(z)$ are the Weyl $m$-functions associated with $H_{ \pm, x}^{D}$ in $L^{2}((x, \pm \infty))$ $\left(H_{x}^{D}=H_{-, x}^{D} \oplus H_{+, x}^{D}\right)$ with $m_{x}^{-}(z)$ being continuous near $\mu_{j}(x)$ while $m_{x}^{+}(z)$ has a first-order pole with a negative residue at $\mu_{j}(x)$. Thus

$$
G(z, x, x) \underset{z \rightarrow \mu_{j}(x)}{=} c\left[z-\mu_{J}(x)\right]+O\left(\left[z-\mu_{j}(x)\right]^{2}\right), \quad \mu_{j}(x) \in\left(0, V_{+}\right)
$$

for some $c>0$ and hence $\xi(\lambda, x)$ has the jump discontinuity

$$
\lim _{\varepsilon \downarrow 0} \xi\left(\mu_{j}(x) \pm \varepsilon\right)=\left\{\begin{array}{l}
0 \\
1
\end{array}\right.
$$

at $\mu_{j}(x) \in\left(0, V_{+}\right)$. A comparison of formulas (3.10) for $\xi(\lambda, x), \lambda>V_{-}$and (3.7) for $R^{l}(\lambda)$ then shows that

$$
1+R^{l}\left(\mu_{j}(x)\right) \frac{f_{-}\left(\mu_{j}(x), x\right)^{2}}{\left|f_{-}\left(\mu_{j}(x), x\right)\right|^{2}}=0,
$$

since $f_{+}\left(\mu_{j}(x), x\right)=0$. Clearly $\xi(\lambda, x)$ is continuous in $\lambda>0$ away from these special energies $\mu_{j}(x) \in\left(0, V_{+}\right]$.

Next, we shall briefly consider the behavior of $\xi(\lambda, x)$ as $\lambda \downarrow 0$ and as $\lambda \downarrow V_{+}$ ( since $H$ changes spectral multiplicity at $V_{+}$) similarly to the discussion at the end of Sect. 2. We shall assume

$$
\int_{-\infty}^{0} d x\left(1+x^{2}\right)|V(x)|+\int_{0}^{\infty} d x\left(1+x^{2}\right)\left|V(x)-V_{+}\right|<\infty
$$

in addition to (3.1) and consider the following case distinctions depending on whether or not $H$ has a threshold resonance at $\lambda=0$.

Case I. $W\left(f_{-}(0), f_{+}(0)\right) \neq 0$ and $f_{-}(0, x) f_{+}(0, x) \neq 0$.

Then, since $f_{ \pm}(0, x)$ are real-valued, one infers

$$
\begin{gathered}
R^{l}(\lambda) \underset{\lambda \downarrow 0}{=}-1+O\left(\lambda^{1 / 2}\right), \\
G(\lambda+i 0, x, x) \underset{\lambda \downarrow 0}{=} \frac{f_{+}(0, x) f_{-}(0, x)}{W\left(f_{+}(0), f_{-}(0)\right)}+O\left(\lambda^{1 / 2}\right),
\end{gathered}
$$

and hence

$$
\xi(\lambda, x)=\pi^{-1} \arg [G(\lambda+i 0, x, x)] \underset{\lambda \downarrow 0}{=} O\left(\lambda^{1 / 2}\right) \text { in case I . }
$$

Case II. $W\left(f_{-}(0), f_{+}(0)\right)=0$ and $f_{-}(0, x) f_{+}(0, x) \neq 0$.

In this case one infers (see, e.g., Proposition 2.4 in [4] or Lemma 2.5 in [13]) that

$$
\begin{aligned}
& W\left(f_{-}(\lambda), f_{+}(\lambda)\right) \underset{\lambda \downarrow 0}{=} i \gamma \lambda^{1 / 2}+O(\lambda), \gamma \in \mathbb{R} \backslash\{0\}, \\
& G(z+i 0, x, x) \underset{\lambda \downarrow 0}{=}(i / \gamma) f_{+}(0, x) f_{-}(0, x)\left[1+O\left(\lambda^{1 / 2}\right)\right] .
\end{aligned}
$$


Thus we get

$$
\xi(\lambda, x)=\pi^{-1} \arg [G(\lambda+i 0, x, x)] \underset{\lambda \downarrow 0}{=} \frac{1}{2}+O\left(\lambda^{1 / 2}\right) \text { in case II } .
$$

Discussion of the case $\lambda \downarrow V_{+}$remains. Since $f_{+}\left(V_{+}, x\right)$ is real-valued and $W\left(f_{-}(\lambda), f_{+}(\lambda)\right) \neq 0$ for $\lambda>0$ (see, e.g., Lemma 1.2 in [4] or Lemma 2.1 in [13]), one infers that

$$
\begin{gathered}
R^{r}(\lambda) \underset{\lambda \downarrow V_{+}}{\bar{V}_{i}}-1+O\left(\left(\lambda-V_{+}\right)^{1 / 2}\right) \\
G(\lambda+i 0, x, x) \underset{\lambda \downarrow V_{+}}{=} \frac{f_{+}\left(V_{+}, x\right) f_{-}\left(V_{+}, x\right)}{W\left(f_{+}\left(V_{+}\right), f_{-}\left(V_{+}\right)\right)}+O\left(\left(\lambda-V_{+}\right)^{1 / 2}\right),
\end{gathered}
$$

and hence

$$
\xi(\lambda, x) \underset{\lambda \downarrow V_{+}}{=} \frac{1}{2}+\pi^{-1} \operatorname{Im}\left\{\ln \left[1+R^{l}\left(V_{+}\right) \frac{f_{-}\left(V_{+}, x\right)^{2}}{\left|f_{-}\left(V_{+}, x\right)\right|^{2}}\right]\right\}+O\left(\left(\lambda-V_{+}\right)^{1 / 2}\right) .
$$

This serves as an illustration that $\xi(\lambda, x)$ is insensitive to the fact that $H$ changes its spectral multiplicity at $V_{+}$.

We conclude this section with a brief discussion of the case where $V(x) \underset{x \rightarrow \infty}{\longrightarrow} \infty$, that is, we now assume

$$
V \in C(\mathbb{R}), \int_{-\infty}^{0} d x|V(x)|<\infty, \quad \lim _{x \rightarrow \infty} V(x)=\infty
$$

$H$ is then defined as the form sum of $H_{0}=-\frac{d^{2}}{d x^{2}}$ and $V$ in $L^{2}(\mathbb{R})$. Then $f_{-}(z, x)$ can be defined as in (3.2) (or (2.13)) and, since $V(x) \rightarrow \infty$ as $x \rightarrow \infty$, the Weyl $m$-function associated with $H_{+, 0}^{D}$ (the restriction of $H$ to $(0, \infty)$ with a Dirichlet boundary condition at $x=0)$ is meromorphic. Hence, there exists an entire function $f_{+}(z, x)$ satisfying

$$
f_{+}(z, .) \in L^{2}((0, \infty)), \quad z \in \mathbb{C}
$$

and (3.3). $f_{+}(z, x)$ can be chosen to be real-valued for $\lambda \in \mathbb{R}$ (see, e.g., [26] for further details). $H$ now has simple spectrum which is purely absolutely continuous on $(0, \infty)$. The reflection coefficient from left incidence is then defined as in (3.4), that is,

$$
R^{l}(\lambda)=-\frac{\overline{W\left(f_{-}(\lambda), f_{+}(\lambda)\right)}}{W\left(f_{-}(\lambda), f_{+}(\lambda)\right)}, \quad \lambda>0
$$

and hence

$$
\left|R^{l}(\lambda)\right|=1, \quad \lambda>0
$$

proves total reflection from left incidence at all positive energies $\lambda>0$. The Green's function $G(z, x, x)$ of $H$ now satisfies

$$
\begin{aligned}
G(\lambda+i 0, x, x) & =\frac{f_{+}(\lambda, x) f_{-}(\lambda, x)}{W\left(f_{+}(\lambda), f_{-}(\lambda)\right)} \\
& =\left(i / 2 \lambda^{1 / 2}\right)\left|f_{-}(\lambda, x)\right|^{2}\left[1+R^{l}(\lambda) \frac{f_{-}(\lambda, x)^{2}}{\left|f_{-}(\lambda, x)\right|^{2}}\right], \quad \lambda>0,
\end{aligned}
$$


and one infers

$$
\xi(\lambda, x)=\frac{1}{2}+\pi^{-1} \operatorname{Im}\left\{\ln \left[1+R^{l}(\lambda) \frac{f_{-}(\lambda, x)^{2}}{\left|f_{-}(\lambda, x)\right|^{2}}\right]\right\}, \quad \lambda>0
$$

as in (3.10). However, due to the total reflection at all positive energies $\lambda>0$,

$$
R^{l}(\lambda)=e^{i r(\lambda)} \underset{\lambda \rightarrow \infty}{\nrightarrow} 0
$$

for some real-valued function $r$ and hence

$$
1-2 \xi(\lambda, x) \underset{\lambda \rightarrow \infty}{\not \rightarrow} 0 .
$$

In fact, in the explicit example $V(x)=e^{x}$ discussed, for instance in [26], where $R^{l}(\lambda)=-\exp \left\{2 i \arg \left[\Gamma\left(1+2 i \lambda^{1 / 2}\right)\right]\right\}, \lambda \geq 0$ (here $\Gamma($.$) denotes the gamma function),$ one can verify that $[1-2 \xi(., x)] \notin L^{1}((0, \infty) ; d \lambda), x \in \mathbb{R}$. As a consequence, the Abelian limit in the trace formula (1.6) for $V(x)$, in general, cannot be removed in the case (3.36) and hence (1.6) represents a genuine summability method in this situation. As mentioned briefly in the introduction, this becomes even more transparent in the case where $V(x) \underset{x \rightarrow \pm \infty}{\rightarrow} \infty$ since then for all $x \in \mathbb{R}$ and a.e. $\lambda \in \mathbb{R},|1-2 \xi(\lambda, x)|=1$.

\section{Hill Operators with Impurities}

In our final section, we shall consider short-range perturbations $W$ of Hill operators $H^{o}=-\frac{d^{2}}{d x^{2}}+V^{o}$, and hence extend the results of Sect. 2 to scattering off impurities (defects) in one-dimensional solids.

Again, most of the results in this section are valid under minimal smoothness assumptions of $V^{o}$ and $W$. However, since our main result in Theorem 4.3 requires a certain regularity of $V^{o}$ and $W$, we shall avoid technicalities and suppose these regularity assumptions throughout this section.

We start by briefly reviewing the necessary Floquet theory associated with the periodic background potential $V^{o}$ satisfying

$$
V^{o} \in H^{1,2}([0, a]), \quad V^{o} \text { real-valued, } \quad V^{o}(x+a)=V^{o}(x), \quad x \in \mathbb{R}
$$

for some $a>0$. The corresponding Hill operator $H^{o}$ in $L^{2}(\mathbb{R})$ is then defined by

$$
H^{o}=-\frac{d^{2}}{d x^{2}}+V^{o}, \quad \mathcal{D}\left(H^{o}\right)=H^{2,2}(\mathbb{R}) .
$$

The spectrum of $H^{o}$ is purely absolutely continuous of the type

$$
\sigma\left(H^{o}\right)=\bigcup_{n \in \mathbb{N}}\left[E_{2(n-1)}^{o}, E_{2 n-1}^{o}\right], E_{0}^{o}<E_{1}^{o} \leq E_{2}^{o}<E_{3}^{o} \leq E_{4}^{o}<E_{5}^{o} \leq \cdots
$$

with uniform multiplicity two on $\sigma\left(H^{o}\right)^{o}$. (We recall that $A^{o}$ denotes the interior of $A \subset \mathbb{R}$.) An entire fundamental system of distributional solutions of

$$
H^{o} \psi(z, x)=z \psi(z, x)
$$


with respect to $z$ is then provided by $s^{o}(z, x)$ and $c^{o}(z, x)$ defined as

$$
\begin{gathered}
s^{o}(z, x)=z^{-1 / 2} \sin \left(z^{1 / 2} x\right)+\int_{0}^{x} d x^{\prime} z^{-1 / 2} \sin \left[z^{1 / 2}\left(x-x^{\prime}\right)\right] V^{o}\left(x^{\prime}\right) s^{o}\left(z, x^{\prime}\right), \\
c^{o}(z, x)=\cos \left(z^{1 / 2} x\right)+\int_{0}^{x} d x^{\prime} z^{-1 / 2} \sin \left[z^{1 / 2}\left(x-x^{\prime}\right)\right] V^{o}\left(x^{\prime}\right) c^{o}\left(z, x^{\prime}\right), \quad z \in \mathbb{C}, \\
W\left(s^{o}(z), c^{o}(z)\right)=-1, \quad z \in \mathbb{C} .
\end{gathered}
$$

The discriminant $\Delta(z)$ and Floquet parameter (Bloch momentum) $\theta(z)$ are then defined by

$$
\begin{aligned}
\Delta(z) & =\left[c^{o}(z, a)+s^{o \prime}(z, a)\right] / 2, \\
e^{ \pm l \theta(z) a} & =\Delta(z) \mp \sqrt{\Delta(z)^{2}-1} .
\end{aligned}
$$

Thus

$$
\cos [\theta(z) a]=\Delta(z), \quad \sin [\theta(z) a]=i \sqrt{\Delta(z)^{2}-1},
$$

and the branch of $\sqrt{ }$. is chosen such that $\sqrt{\Delta(\lambda)^{2}-1}>0$ for $\lambda<E_{0}^{o}$ and hence

$$
\begin{gathered}
-i \theta(\lambda)>0, \lambda<E_{0}^{o}, \quad \theta(\lambda) \in \mathbb{R} \Longleftrightarrow \lambda \in \sigma\left(H^{o}\right), \\
-i \theta(\lambda) \in(0, \infty) \Longleftrightarrow \lambda \in \mathbb{R} \backslash \sigma\left(H^{o}\right) .
\end{gathered}
$$

The Floquet solutions of $H^{o}$ are defined by

$$
\begin{aligned}
f_{ \pm}^{o}(z, x) & =c^{o}(z, x)+s^{o}(z, x)\left[\Delta(z) \mp \sqrt{\Delta(z)^{2}-1}-c^{o}(z, a)\right] / s^{o}(z, a) \\
& =e^{ \pm \imath \theta(z) x} p_{ \pm}(z, x), \quad p_{ \pm}(z, x+a)=p_{ \pm}(z, x) .
\end{aligned}
$$

They satisfy

$$
\begin{aligned}
& f_{ \pm}^{o}(z, .) \in L^{2}((R, \pm \infty)), \quad R \in \mathbb{R}, \quad z \in \mathbb{C} \backslash \sigma\left(H^{o}\right), \\
& f_{-}^{o}(\lambda, x)=\overline{f_{+}^{o}(\lambda, x)}, \quad \lambda \in \sigma\left(H^{o}\right), \\
& f_{ \pm}^{o}(\lambda, x) \text { are real-valued for } \lambda \in \mathbb{R} \backslash \sigma\left(H^{o}\right)^{o} \text {, } \\
& W\left(f_{-}^{o}(z), f_{+}^{o}(z)\right)=-2 \sqrt{\Delta(z)^{2}-1} / s^{o}(z, a), \\
& W\left(f_{\mp}^{o}(\lambda), \overline{f_{\mp}^{o}(\lambda)}\right)=\left\{\begin{array}{l} 
\pm 2 i \sin [\theta(\lambda) a] / s^{o}(\lambda, a), \lambda \in \sigma\left(H^{o}\right)^{o} \\
0, \lambda \in \mathbb{R} \backslash \sigma\left(H^{o}\right)^{o} .
\end{array}\right.
\end{aligned}
$$

For details in connection with (4.3)-(4.16), see, for example, [9, 10, 28], Sect. 7.4, [29], Sect. 3.4, [36, 37], and the references therein.

If $H_{y}^{o, D}$ denotes the associated Dirichlet operator in $L^{2}(\mathbb{R})$ defined analogously to (2.7) with spectrum

$$
\begin{gathered}
\sigma\left(H_{y}^{o, D}\right)=\left\{\mu_{n}^{o}(y)\right\}_{n \in \mathbb{N}} \cup \sigma\left(H^{o}\right), \quad \sigma_{\mathrm{ess}}\left(H_{y}^{o, D}\right)=\sigma\left(H^{o}\right), \\
E_{2 n-1}^{o} \leq \mu_{n}^{o}(y) \leq E_{2 n}^{o}, \quad n \in \mathbb{N},
\end{gathered}
$$


the trace formula (1.6) applied to $V^{o}(x)$ yields (see $\left.[16,19]\right)$

$$
\begin{aligned}
V^{o}(x) & =E_{0}^{o}+\lim _{\varepsilon \downarrow 0} \int_{E_{0}^{o}}^{\infty} d \lambda e^{-\varepsilon \lambda}\left[1-2 \xi^{o}(\lambda, x)\right] \\
& =E_{0}^{o}+\sum_{n=1}^{\infty}\left[E_{2 n-1}^{o}+E_{2 n}^{o}-2 \mu_{n}^{o}(x)\right] .
\end{aligned}
$$

Here $\pi \xi^{o}(\lambda, x)$ denotes the argument of the Green's function $G^{o}(z, x, x)$ of $H^{o}$ (see (2.24)) and one explicitly obtains

$$
\xi^{o}(\lambda, x)=\left\{\begin{array}{l}
1, E_{2 n-1}^{o}<\lambda<\mu_{n}^{o}(x) \\
0, \mu_{n}^{o}(x)<\lambda<E_{2 n}^{o} \\
\frac{1}{2}, E_{2(n-1)}^{o}<\lambda<E_{2 n-1}^{o} .
\end{array}\right.
$$

Moreover, the assumption $V^{o} \in H^{1,2}([0, a])$ implies the finiteness of the total gap length (see, e.g., [23] or Theorem 1.5.2 in [29])

$$
\sum_{n=1}^{\infty}\left|E_{2 n}^{o}-E_{2 n-1}^{o}\right|<\infty
$$

and this has been used to infer (4.19) from (4.18) and (4.20).

Next we briefly turn to the impurity potential $W$ assuming

$$
W \in H^{2,1}(\mathbb{R}), \quad W \text { real-valued, } \quad W \in L^{1}(\mathbb{R} ;(1+|x|) d x) .
$$

The total Hamiltonian $H$ in $L^{2}(\mathbb{R})$ is then defined by

$$
H=-\frac{d^{2}}{d x^{2}}+V, \quad \mathcal{D}(H)=H^{2,2}(\mathbb{R}), \quad V(x)=V^{o}(x)+W(x) .
$$

The spectrum $\sigma(H)$ of $H$ is now of the type

$$
\sigma(H)=\sigma_{p}(H) \cup \sigma\left(H^{o}\right), \quad \sigma_{\mathrm{ess}}(H)=\sigma\left(H^{o}\right),
$$

where the point spectrum $\sigma_{p}(H)$ (the set of eigenvalues) of $H$ may be denoted by

$$
\sigma_{p}(H)=\bigcup_{n=0}^{\infty} \sigma_{p, n}
$$

$$
\sigma_{p, 0}=\left\{e_{0, j}\right\}_{j \in J_{0}} \subset\left(-\infty, E_{0}^{o}\right), \quad \sigma_{p, n}=\left\{e_{n, j}\right\}_{j \in J_{n}} \subset\left(E_{2 n-1}^{o}, E_{2 n}^{o}\right), \quad e_{n, j}<e_{n, j+1}
$$

with

$$
J_{n}=\left\{\begin{array}{l}
\emptyset \\
\left\{0,1,2, \ldots, N_{n}\right\}
\end{array}, \quad n \in \mathbb{N}_{0},\right.
$$

an appropriate index set. Similarly to Sect. 2, we shall also need the notation

$$
\begin{gathered}
J_{0,+}=\left\{\begin{array}{l}
\emptyset \\
\left\{1,2, \ldots, N_{0}, N_{0}+1\right\}
\end{array}, \quad J_{n,+}=\left\{\begin{array}{l}
\emptyset \\
\left\{0,1,2, \ldots, N_{n}, N_{n}+1\right\}
\end{array}, \quad n \in \mathbb{N},\right.\right. \\
e_{0, N_{0}+1}=E_{0}^{o}, \quad e_{n,-1}=E_{2 n-1}^{o}, \quad e_{n, N_{n}+1}=E_{2 n}^{o},
\end{gathered}
$$


depending on whether $J_{n}$ is empty or finite. Each eigenvalue of $H$ is simple, $\sigma_{p}(H) \cap \sigma\left(H^{o}\right)=\emptyset$, and the spectrum of $H$ in $\sigma\left(H^{o}\right)^{o}$ is purely absolutely continuous and of uniform multiplicity two under hypotheses (4.1) and (4.22).

Remark 4.1. We have chosen to add the hypothesis $W \in L^{1}(\mathbb{R} ;(1+|x|) d x)$ from the outset in (4.22) since it guarantees finiteness of the discrete spectrum $\sigma_{p, n}$ of $H$ in any of its essential spectral gaps $\left(-\infty, E_{0}^{o}\right),\left(E_{2 n-1}^{o}, E_{2 n}^{o}\right)$ as proven in [31]. Moreover, it can be shown that $H$ has at most two eigenvalues in $\left(E_{2 n-1}^{o}, E_{2 n}^{o}\right)$ for $n$ sufficiently large (i.e., $N_{n} \leq 2$ for $n$ large enough), and if $\int_{\mathbb{R}} d x W(x) \neq 0$ precisely one eigenvalue in $\left(E_{2 n-1}^{o}, E_{2 n}^{o}\right)$ for $n$ sufficiently large (i.e., $N_{n}=1$ if $\int_{\mathbb{R}} d x W(x) \neq$ 0 for $n$ large enough), see [9, 15, 32, 36, 37]. The following material can be developed without the assumption $W \in L^{1}(\mathbb{R} ;(1+|x|) d x)$ but only at the expense of introducing a considerably more involved bulk of notations since elements of $\sigma_{p, n}$ may then accumulate at $\left(E_{2 n-1}^{o}\right.$ and/or $\left.E_{2 n}^{o}\right)$.

Next we briefly introduce the associated Dirichlet operator $H_{y}^{D}$ in $L^{2}(\mathbb{R})$ defined as in (2.7). Its spectrum is then given by

$$
\sigma\left(H_{y}^{D}\right)=\sigma_{p}\left(H_{y}^{D}\right) \cup \sigma\left(H^{o}\right), \quad \sigma_{\mathrm{ess}}\left(H_{y}^{D}\right)=\sigma\left(H^{o}\right),
$$

where

$$
\begin{gathered}
\sigma_{p}\left(H_{y}^{D}\right)=\bigcup_{n=0}^{\infty} \sigma_{p, n}^{D}(y), \\
\sigma_{p, 0}^{D}(y)=\left\{\mu_{0, j}(y)\right\}_{j \in J_{0},+} \cap\left(e_{0,0}, E_{0}^{o}\right), \\
e_{0,0}<\mu_{0,1}(y) \leq e_{0,1}, \quad e_{0, j-1} \leq \mu_{0, j}(y) \leq e_{0, j}, \quad j \in J_{0,+} \backslash\{1\}, \\
\sigma_{p, n}^{D}(y)=\left\{\mu_{n, j}(y)\right\}_{j} \in J_{n,+} \cap\left(E_{2 n-1}^{o}, E_{2 n}^{o}\right), \quad n \in \mathbb{N}, \\
e_{n, j-1} \leq \mu_{n, j}(y) \leq e_{n, j}, \quad j \in J_{n,+}, \quad n \in \mathbb{N} .
\end{gathered}
$$

(Our notation in (4.30), (4.31) indicates that the limiting cases $\mu_{n, 0}(y)=e_{0,-1}=$ $E_{2 n-1}^{o}, n \in \mathbb{N}, \mu_{n, N_{n}+1}(y)=e_{n, N_{n}+1}=E_{2 n}^{o}, n \in \mathbb{N}$ are not Dirichlet eigenvalues since $H$ has no $L^{2}((y, \pm \infty))$ eigenfunctions at $E_{n}^{o}, n \in \mathbb{N}_{0}$.) The spectrum of $H_{y}^{D}$ in $\sigma\left(H^{o}\right)^{o}$ is purely absolutely continuous and of uniform multiplicity two. Similarly to Sect. 2, $\mu_{n, j}(y)$ is a simple eigenvalue of $H_{y}^{D}$ if and only if $e_{n, j-1}<\mu_{n, j}(y)<e_{n, j}$, whereas if $\mu_{n, j}(y) \in\left\{e_{n, j-1}, e_{n, j}\right\}$, then $\mu_{n, j}(y)$ has multiplicity two (excluding the cases $\left.\mu_{n, j}(y)=E_{2 n-1}^{o}, E_{2 n}^{o}\right)$. For details in the context of (4.24)-(4.31) see, for example, $[9,10,15,31,32,36,37]$, and the references therein.

Impurity (defect) scattering associated with the pair $\left(H, H^{o}\right)$ can then be summarized as follows (see, e.g., [9, 10, 13], and the references therein). The Jost solutions $f_{ \pm}(z, x)$ of $H$ are defined by

$$
\begin{gathered}
f_{ \pm}(z, x)=f_{ \pm}^{o}(z, x)-\int_{x}^{ \pm \infty} d x^{\prime} g^{o}\left(z, x, x^{\prime}\right) W\left(x^{\prime}\right) f_{ \pm}\left(z, x^{\prime}\right), z \in \mathbb{C} \backslash\left\{E_{n}^{o}\right\}_{n \in \mathbb{N}_{0}}, \\
g^{o}\left(z, x, x^{\prime}\right)=\left[f_{+}^{o}(z, x) f_{-}^{o}\left(z, x^{\prime}\right)-f_{+}^{o}\left(z, x^{\prime}\right) f_{-}^{o}(z, x)\right] / W\left(f_{-}^{o}(z), f_{+}^{o}(z)\right),
\end{gathered}
$$

such that

$$
H f_{ \pm}(z, x)=z f_{ \pm}(z, x), \quad z \in \mathbb{C} \backslash\left\{E_{n}^{o}\right\}_{n \in \mathbb{N}_{0}}
$$


in the distributional sense. The unitary scattering matrix $S(\lambda), \lambda \in \sigma\left(H^{o}\right)^{o}$ in $\mathbb{C}^{2}$ associated with the pair $\left(H, H^{\circ}\right)$ then reads as follows:

$$
\begin{aligned}
& S(\lambda)=\left(\begin{array}{cc}
T(\lambda) & R^{r}(\lambda) \\
R^{l}(\lambda) & T(\lambda)
\end{array}\right), \quad \lambda \in \sigma\left(H^{o}\right)^{o}, \\
& \left(T(\lambda)=\frac{2 i \sin [\theta(\lambda) a] / s^{o}(\lambda, a)}{W\left(f_{-}(\lambda), f_{+}(\lambda)\right)}=\left\{1-\frac{s^{o}(\lambda, a)}{2 i \sin [\theta(\lambda) a]_{\mathbb{R}}} \int_{\mathbb{R}} d x W(x) f_{ \pm}^{o}(\lambda, x) f_{\mp}(\lambda, x)\right\}^{-1},\right. \\
& R^{l}(\lambda)=-\frac{W\left(\overline{f_{-}(\lambda)}, f_{+}(\lambda)\right)}{W\left(f_{-}(\lambda), f_{+}(\lambda)\right)}=\frac{T(\lambda) s^{o}(\lambda, a)}{2 i \sin [\theta(\lambda) a]_{\mathbb{R}}} \int_{\mathbb{R}} d x W(x) f_{+}^{o}(\lambda, x) f_{+}(\lambda, x), \\
& R^{r}(\lambda)=-\frac{W\left(f_{-}(\lambda), \overline{f_{+}(\lambda)}\right)}{W\left(f_{-}(\lambda), f_{+}(\lambda)\right)}=\frac{T(\lambda) s^{o}(\lambda, a)}{2 i \sin [\theta(\lambda) a]_{\mathbb{R}}} \int_{\mathbb{R}} d x W(x) f_{-}^{o}(\lambda, x) f_{-}(\lambda, x), \\
& G(\lambda+i 0, x, x)=\frac{f_{+}(\lambda, x) f_{-}(\lambda, x)}{W\left(f_{+}(\lambda), f_{-}(\lambda)\right)} \\
& =\frac{i s^{o}(\lambda, a)}{2 \sin [\theta(\lambda) a]}\left|f_{ \pm}(\lambda, x)\right|^{2}\left[1+R^{l}(\lambda) \frac{f_{ \pm}(\lambda, x)^{2}}{\left|f_{ \pm}(\lambda, x)\right|^{2}}\right], \lambda \in \sigma\left(H^{o}\right)^{o}, x \in \mathbb{R} .
\end{aligned}
$$

Since $V=V^{o}+W$ is continuous and bounded from below, relations (2.20)(2.27), in particular, the definition (2.24) of $\xi(\lambda, x)$ and the trace formula (2.27), are valid.

In attempting to generalize Lemma 2.1, however, one is faced with the following problem. Although

$$
\begin{gathered}
s^{o}(\lambda, a) \underset{\lambda \rightarrow \infty}{=} \lambda^{-1 / 2} \sin \left(\lambda^{1 / 2} a\right)-(2 \lambda)^{-1} \cos \left(\lambda^{1 / 2} a\right) \int_{0}^{a} d x V^{o}(x)+O\left(\lambda^{-3 / 2}\right) \\
\theta(\lambda) \underset{\lambda \rightarrow \infty}{=} \lambda^{1 / 2}+O(1)
\end{gathered}
$$

and hence

$$
T(\lambda) \underset{\lambda \rightarrow \infty}{\rightarrow} 1, \quad R^{r(l)}(\lambda) \underset{\lambda \rightarrow \infty}{\rightarrow} 0 \text { pointwise for } \lambda \in \sigma\left(H^{o}\right)^{o}
$$

that is, away from the essential spectral band edges $\left\{E_{n}^{o}\right\}_{n \in \mathbb{N}_{0}}$ of $H$, the factors $\sin [\theta(\lambda) a]$ in the denominators of (4.36)-(4.38) prevent the convergence in (4.42) at the band edges. In fact, as we will briefly explore at the end of this section, one generally has $R^{l, r}\left(E_{n}^{o}\right)=-1$, and hence a result such as $R^{l(r)}(\lambda) \underset{\lambda \rightarrow \infty}{=} o\left(\lambda^{-3 / 2}\right)$ in (2.36) is usually false in the present impurity scattering situation. Nevertheless, by separately considering sufficiently small compact intervals $\sigma_{n} \subset \sigma\left(H^{o}\right)$ with $E_{n}^{o} \in \partial \sigma_{n}$ and the remaining spectral band $\left[E_{2(n-1)}^{o}, E_{2 n-1}^{o}\right] \backslash\left\{\sigma_{2 n-1} \cup \sigma_{2 n}\right\}$, a device studied in detail by Firsova $[9,10]$, we will be able to prove a suitable analog of Lemma 2.1 . 
Lemma 4.2 Suppose $V=V^{o}+W$, where $V^{o} \in H^{1,2}([0, a])$ is real-valued, $V^{o}(x+$ $a)=V^{o}(x)$ for some $a>0, W \in H^{2,1}(\mathbb{R})$ is real-valued, and $W \in L^{1}(\mathbb{R} ;(1+$ $|x|) d x)$. Then for all $x \in \mathbb{R}$,

$$
\begin{gathered}
\xi(\lambda, x)=0, \quad \lambda<E_{o}=\inf \sigma(H), \\
\xi(\lambda, x)=\frac{1}{2}+\pi^{-1} \operatorname{Im}\left\{\ln \left[1+R^{l}(\dot{\lambda}) \frac{f_{ \pm}(\lambda, x)^{2}}{\left|f_{ \pm}(\lambda, x)\right|^{2}}\right]\right\}, \quad \lambda \in \sigma\left(H^{o}\right),
\end{gathered}
$$

and $\xi(\lambda, x)$ is continuous for $\lambda \in \sigma\left(H^{o}\right)^{o}$. Moreover,

$$
|1-2 \xi(\lambda, x)| \leq\left|R^{r(l)}(\lambda)\right|, \quad \lambda \in \sigma\left(H^{o}\right)^{o} .
$$

In addition, there exist compact intervals $\sigma_{n} \subset \sigma\left(H^{o}\right), E_{n}^{o} \in \partial \sigma_{n}, n \in \mathbb{N}_{0}$ with $\sum_{n \in \mathbb{N}_{0}}\left|\sigma_{n}\right|<\infty(|\cdot|$ denoting Lebesgue measure) such that

$$
[1-2 \xi(\lambda, x)] \underset{\lambda \rightarrow \infty}{=} o\left(\lambda^{-3 / 2}\right) \text { for } \lambda \in \sigma\left(H^{o}\right) \backslash \bigcup_{n \in \mathbb{N}_{0}} \sigma_{n}
$$

uniformly with respect to $x \in \mathbb{R}$. In addition,

$$
R^{r(l)} \in L^{1}\left(\sigma\left(H^{o}\right) ; d \lambda\right) .
$$

Finally, we note that

$$
\begin{aligned}
& \xi(\lambda, x)=\left\{\begin{array}{l}
0, \lambda<e_{0,0}, \mu_{n, j}(x)<\lambda<e_{n, j} \\
1, e_{n, j-1}<\lambda<\mu_{n, j}(x)
\end{array} \text { if } \mu_{n, j}(x) \in\left(e_{n, j-1}, e_{n, j}\right),\right. \\
& \xi(\lambda, x)=\left\{\begin{array}{l}
0, e_{n, j-1}<\lambda<e_{n, j} \text { if } \mu_{n, j}(x)=e_{n, j-1} \\
1, e_{n, j-1}<\lambda<e_{n, j} \text { if } \mu_{n, j}(x)=e_{n, j}
\end{array}\right.
\end{aligned}
$$

whenever $\sigma_{p, n}(H) \neq \emptyset$.

Proof. Equations (4.43), (4.48) and (4.49) reflect the general behavior of $\xi(\lambda, x)$ in spectral gaps of $H$ since $G(z, x, x)>0$ for $z<\inf \sigma(H)$ and $G(z, x, x)$ is real-valued for $z$ in any (non-empty) spectral gap of $H$. Equation (4.44) follows from (2.24) and (4.39) (we note that $s^{o}(\lambda, a)$ is real-valued for $\lambda \in \mathbb{R}$ and $\theta(\lambda) \in \mathbb{R}$ for $\lambda \in \sigma\left(H^{o}\right)$ by (4.10)). Since $G(\lambda+i 0, x, x)$ is continuous and zero-free for $\lambda \in \sigma\left(H^{o}\right)^{o}, \xi(\lambda, x)$ is continuous in $\lambda \in \sigma\left(H^{o}\right)$. Inequality (4.45) is then clear from (4.44). For the explicit construction of the compact intervals $\sigma_{n}$ with $E_{n}^{o} \in \partial \sigma_{n}, \sum_{n \in \mathbb{N}_{0}}\left|\sigma_{n}\right|<\infty$ such that

$$
R^{r(l)}(\lambda) \underset{\lambda \rightarrow \infty}{=} o\left(\lambda^{-1 / 2}\right) \quad \text { for } \lambda \in \sigma\left(H^{o}\right) \backslash \bigcup_{n \in \mathbb{N}_{0}} \sigma_{n},
$$

we refer to $[9,10]$. Here we only mention that $(4.50)$ is implied by the asymptotic relation (4.41) and, assuming $\lambda \in \sigma\left(H^{o}\right) \backslash \bigcup_{n \in \mathbb{N}_{0}} \sigma_{n}$, by

$$
\begin{aligned}
& W\left(f_{-}^{o}(\lambda), f_{+}^{o}(\lambda)\right)=\frac{2 i \sin [\theta(\lambda) a]}{s^{o}(\lambda, a)} \underset{\lambda \rightarrow \infty}{=} 2 i \lambda^{1 / 2}\left[1+O\left(|\lambda|^{-1 / 8}\right)\right], \\
& T(\lambda) \underset{\lambda \rightarrow \infty}{=} 1+\left(1 / 2 i \lambda^{1 / 2}\right)\left[\int_{\mathbb{R}} d x W(x)+O\left(|\lambda|^{-1 / 8}\right)\right], \\
& R^{r}(\lambda) \underset{\substack{r \\
\lambda \rightarrow \infty}}{=}\left(1 / 2 i \lambda^{1 / 2}\right)\left[\int_{\mathbb{R}} d x W(x) e^{\mp 2 i \theta(\lambda) x}+O\left(|\lambda|^{-1 / 8}\right)\right]
\end{aligned}
$$


as proven in [9] (see also [10]). In particular, in order to arrive at (4.52), (4.53) one combines (4.41), (4.51), (4.36)-(4.38), and

$$
\begin{gathered}
p_{ \pm}(\lambda, x) \underset{\lambda \rightarrow \infty}{=} 1+O\left(|\lambda|^{-1 / 8}\right), \quad \lambda \in \sigma\left(H^{o}\right) \backslash \bigcup_{n \in N_{0}} \sigma_{n}, \\
\left|f_{ \pm}(\lambda, x)\right| \leqq C(1+|x|), \quad \lambda \in \sigma\left(H^{o}\right), \\
\left|f_{ \pm}(\lambda, x)-f_{ \pm}^{o}(\lambda, x)\right| \leqq C(1+|x|)(1+|\lambda|)^{-1 / 2}, \quad \lambda \in \sigma\left(H^{o}\right) .
\end{gathered}
$$

Here $p_{ \pm}(\lambda, x)$ has been introduced in (4.11), and (4.55) and (4.56) follow from (4.32), (4.33) (see $[9,10])$. Analogous relations for the first and second $x$ derivatives of $p_{ \pm}(\lambda, x)$ and $f_{ \pm}(\lambda, x)$ then yield

$$
R^{r(l)}(\lambda) \underset{\lambda \rightarrow \infty}{=} o\left(\lambda^{-3 / 2}\right), \quad \lambda \in \sigma\left(H^{o}\right) \backslash \bigcup_{n \in N_{0}} \sigma_{n}
$$

after two integrations by parts in (4.37), (4.38). Together with (4.45) this proves (4.46). Using $\left|R^{r(l)}(\lambda)\right| \leqq 1, \lambda \in \sigma\left(H^{o}\right)$ (as a consequence of the unitarity of the scattering matrix (4.35)), one then infers (4.47) from

$$
\int_{\sigma\left(H^{o}\right)} d \lambda\left|R^{r(l)}(\lambda)\right| \leqq \int_{n \in N_{0}} \sigma_{n} d \lambda+\int_{\sigma\left(H^{o}\right) \backslash_{n \in N_{0}} \sigma_{n}}\left|o\left(\lambda^{-3 / 2}\right)\right|<\infty .
$$

As in the previous sections, Lemma 4.2 will enable us to remove the Abelian limit in the trace formula (2.27) for $V(x)$ and state the principal result of this section. (We recall our notational conventions in (4.3), (4.17), (4.25)-(4.31).)

Theorem 4.3. Suppose $V=V^{o}+W$, where $V^{o} \in H^{1,2}([0, a])$ is real-valued, $V^{o}(x+a)=V^{o}(x)$ for some $a>0, W \in H^{2,1}(\mathbb{R})$ is real-valued, and $W \in$ $L^{1}(\mathbb{R} ;(1+|x|) d x)$. Let $E_{o}=\inf \sigma(H)$. Then $[1-2 \xi(., x)] \in L^{1}\left(\left(E_{o}, \infty\right) ; d \lambda\right)$, $x \in \mathbb{R}$ and

$$
\begin{aligned}
V(x)= & V^{o}(x)+W(x)=E_{o}+\int_{E_{o}}^{\infty} d \lambda[1-2 \xi(\lambda, x)] \\
= & \left\{2 e_{0,0}+2 \sum_{j \in J_{0,+}}\left[e_{0, j}-\mu_{0, j}(x)\right]-E_{0}^{o}\right\} \\
& +\sum_{n=1}^{\infty}\left\{E_{2 n-1}^{0}+2 \sum_{j \in J_{n,+}}\left[e_{n, j}-\mu_{n, j}(x)\right]-E_{2 n}^{o}\right\}+\int_{\sigma\left(H^{o}\right)} d \lambda[1-2 \xi(\lambda, x)] \\
= & \left\{2 e_{0,0}+2 \sum_{j \in J_{0,+}}\left[e_{0, j}-\mu_{0, j}(x)\right]-E_{0}^{o}\right\} \\
& +\sum_{n=1}^{\infty}\left\{E_{2 n-1}^{0}+2 \sum_{j \in J_{n,+}}\left[e_{n, j}-\mu_{n, j}(x)\right]-E_{2 n}^{o}\right\} \\
& -(2 / \pi) \int_{\sigma\left(H^{o}\right)} d \lambda \operatorname{Im}\left\{\ln \left[1+R^{r}(\lambda) \frac{f_{ \pm}(\lambda, x)^{2}}{\left|f_{ \pm}(\lambda, x)\right|^{2}}\right]\right\}, \quad x \in \mathbb{R} .
\end{aligned}
$$


Similarly,

$$
\begin{aligned}
W(x)= & 2\left\{e_{0,0}+\sum_{j \in J_{0,+}}\left[e_{0, j}-\mu_{0, j}(x)\right]-E_{0}^{o}\right\} \\
& +2 \sum_{n=1}^{\infty}\left\{\mu_{n}^{0}(x)+\sum_{j \in J_{0,+}}\left[e_{n, j}-\mu_{n, j}(x)\right]-E_{2 n}^{o}\right\}+\int_{\sigma\left(H^{o}\right)} d \lambda[1-2 \xi(\lambda, x)] \\
= & 2\left\{e_{0,0}+\sum_{j \in J_{0,+}}\left[e_{0, j}-\mu_{0, j}(x)\right]-E_{0}^{o}\right\} \\
& +2 \sum_{n=1}^{\infty}\left\{\mu_{n}^{0}(x)+\sum_{j \in J_{n,+}}\left[e_{n, j}-\mu_{n, j}(x)\right]-E_{2 n}^{o}\right\} \\
& -(2 / \pi) \int_{\sigma\left(H^{o}\right)} d \lambda \operatorname{Im}\left\{\ln \left[1+R^{l}(\lambda) \frac{f_{ \pm}(\lambda, x)^{2}}{\left|f_{ \pm}(\lambda, x)\right|^{2}}\right]\right\}, \quad x \in \mathbb{R} .
\end{aligned}
$$

If $\sigma_{p, n}(H)=\emptyset$, the corresponding expression $\{\ldots\}$ in (4.60), (4.61) is to be replaced by $\left\{E_{2 n-1}^{o}-2 \mu_{n, 0}(x)+E_{2 n}^{o}\right\}$ if $n \in \mathbb{N}$ and deleted if $n=0$. Similarly, if $\sigma_{p, n}(H)=\emptyset$, the corresponding expression $2\{\ldots\}$ in (4.62), (4.63) is to be replaced by $2\left\{\mu_{n}^{o}(x)-\mu_{n, 0}(x)\right\}$ if $n \in \mathbb{N}$ and deleted if $n=0$.

Proof. The trace formula (4.53) follows from (1.6), (4.45)-(4.47), and the Lebesgue dominated convergence theorem. Equalities (4.54) and (4.55) are then clear from (4.59), (4.44), (4.48), and (4.49). Equations (4.62) and (4.63) are obtained by combining (4.60), (4.61) and (4.19) observing the finite total gap length (4.21).

We note that the analog of Remark 2.2 clearly holds in the present context. Moreover, the threshold behavior of $\xi(\lambda, x)$ in (2.43)-(2.51) near $\lambda=0$ extends to the essential spectral band edges $\left\{E_{n}^{o}\right\}_{n \in N_{0}}$ of $H$ in the current impurity scattering situation. In particular, assuming

$$
W \in L^{1}\left(\mathbb{R} ;\left(1+x^{2}\right) d x\right)
$$

in addition to (4.22), one again distinguishes two cases depending on whether or not $H$ has a threshold resonance at $E_{n}^{o}$.

Case I. $W\left(f_{-}\left(E_{n}^{o}\right), f_{+}\left(E_{n}^{o}\right)\right) \neq 0$ and $f_{-}\left(E_{n}^{o}, x\right) f_{+}\left(E_{n}^{o}, x\right) \neq 0$.

Then

$$
R^{r(l)}\left(E_{n}^{o}\right)=-1
$$

and

$$
\xi(\lambda, x) \underset{\substack{\lambda \rightarrow E_{n}^{o} \\ \lambda \in \sigma\left(H^{o}\right)}}{=} O\left(\left|\lambda-E_{n}^{o}\right|^{1 / 2}\right) \text { in case I . }
$$

Case II. $W\left(f_{-}\left(E_{n}^{o}\right), f_{+}\left(E_{n}^{o}\right)\right)=0$ and $f_{-}\left(E_{n}^{o}, x\right) f_{+}\left(E_{n}^{o}, x\right) \neq 0$.

Then one can show that

$$
\xi(\lambda, x) \underset{\substack{\lambda \rightarrow E_{n}^{o} \\ \lambda \in \sigma\left(H^{o}\right)}}{=} \frac{1}{2}+O\left(\left|\lambda-E_{n}^{o}\right|^{1 / 2}\right) \text { in case II . }
$$


Acknowledgements. We would like to thank Z. Zhao for numerous helpful discussions. F.G. is indebted to the Department of Mathematical Sciences of the University of Trondheim, Norway, and the Department of Mathematics at Caltech, Pasadena, CA for the hospitality extended to him in the summer of 1993. Support by the Norwegian Research Council (NFR) and by Caltech is gratefully acknowledged. H.H. is grateful to the Norwegian Research Council (NFR) for support.

\section{References}

1. Aronszajn, N., Donoghue, W.F.: On exponential representations of analytic functions in the upper half-plane with positive imaginary part. J. Anal. Math. 5, 321-388 (1957)

2. Bollé, D., Gesztesy, F., Wilk, S.F.J.: A complete treatment of low-energy scattering in one dimension. J. Operator Theory 13, 3-31 (1985)

3. Bollé, D., Gesztesy, F., Klaus, M.: Scattering theory for one-dimensional systems with $\int d x V(x)=0$. J. Math. Anal. Appl. 122, 496-518 (1987)

4. Cohen, A., Kappeler, T.: Scattering and inverse scattering for steplike potentials in the Schrödinger equation. Indiana Univ. Math. J. 34, 127-180 (1985)

5. Craig, W.: The trace formula for Schrödinger operators on the line. Commun. Math. Phys. 126, 379-407 (1989)

6. Davies, E.B., Simon, B.: Scattering theory for systems with different spatial asymptotics to the left and right. Commun. Math. Phys. 63, 277-301 (1978)

7. Deift, P., Trubowitz, E.: Inverse scattering on the line. Commun. Pure Appl. Math. 32, 121-251 (1979)

8. Dubrovin, B.A.: Periodic problems for the Korteweg-de Vries equation in the class of finite band potentials. Funct. Anal. Appl. 9, 215-223 (1975)

9. Firsova, N.E.: Levinson formula for perturbed Hill operator. Theoret. Math. Phys. 62, 130-140 (1985)

10. Firsova, N.E.: The direct and inverse scattering problems for the one-dimensional perturbed Hill operator. Math. USSR Sbornik 58, 351-388 (1987)

11. Flaschka, H.: On the inverse problem for Hill's operator. Arch. Rat. Mech. Anal. 59, 293-309 (1975)

12. Gel'fand, I.M., Levitan, B.M.: On a simple identity for eigenvalues of a second order differential operator. Dokl. Akad. Nauk SSSR 88, 593-596 (1953) (Russian); English transl. in Gelfand, Izrail M., Collected papers Vol. I (Gindikin, S.G., Guillemin, V.W., Kirillov, A.A., Kostant, B., Sternberg, S., eds.) Berlin, Heidelberg, New York: Springer, 1987, pp. 457-461

13. Gesztesy, F.: Scattering theory for one-dimensional systems with nontrivial spatial asymptotics. Schrödinger Operators, Aarhus 1985 (Balslev, E., ed.), Lecture Notes in Math. Vol. 1218, Berlin, Heidelberg, New York: Springer, 1986, pp. 93-122

14. Gesztesy, F.: New trace formulas for Schrödinger operators. Evolution Equations. (Ferreyra, G., Ruiz Goldstein, G., Neubrander, F., eds.), New York, Marcel Dekker, 1995, pp. 201-221

15. Gesztesy, F., Simon, B.: A short proof of Zheludev's theorem. Trans. Am. Math. Soc. 335, 329-340 (1993)

16. Gesztesy, F., Simon, B.: The xi function. Acta. Math. (To appear)

17. Gesztesy, F., Holden, H., Simon, B., Zhao, Z.: A trace formula for multidimensional Schrödinger operators. Preprint

18. Gesztesy, F., Holden, H., Simon, B., Zhao, Z.: Trace formulae and inverse spectral theory for Schrödinger operators. Bull. Am. Math. Soc. 29, 250-255 (1993)

19. Gesztesy, F., Holden, H., Simon, B., Zhao, Z.: Higher order trace relations for Schrödinger operators. Rev. Math. Phys. (To appear)

20. Gesztesy, F., Karwowski, W., Zhao, Z.: New types of soliton solutions. Bull. Am. Math. Soc. 27, 266-272 (1992)

21. Gesztesy, F., Karwowski, W., Zhao, Z.: Limits of soliton solutions. Duke Math. J. 68, 101-150 (1992)

22. Hochstadt, H.: On the determination of a Hill's equation from its spectrum. Arch. Rat. Mech. Anal. 19, 353-362 (1965)

23. Iwasaki, K.: Inverse problem for Sturm-Liouville and Hill equation. Ann. Mat. Pura Appl. Ser. 4, 149, 185-206 (1987) 
24. Kotani, S., Krishna, M.: Almost periodicity of some random potentials. J. Funct. Anal. 78, 390-405 (1988)

25. Krein, M.G.: Perturbation determinants and a formula for the traces of unitary and self-adjoint operators. Sov. Math. Dokl. 3, 707-710 (1962)

26. Kristensson, G.: The one-dimensional inverse scattering problem for an increasing potential, J. Math. Phys. 27, 804-815 (1986)

27. Levitan, B.M.: On the closure of the set of finite-zone potentials, Math. USSR Sbornik 51, 67-89 (1985)

28. Levitan, B.M.: Inverse Sturm-Liouville Problems. Utrecht: VNU Science Press, 1987

29. Marchenko, V.A.: Sturm-Liouville Operators and Applications. Basel: Birkhäuser, 1986

30. McKean, H.P., van Moerbeke, P.: The spectrum of Hill's equation. Invent. Math. 30, 217-274 (1975)

31. Rofe-Beketov, F.S.: A test for the finiteness of the number of discrete levels introduced into gaps of a continuous spectrum by perturbations of a periodic potential. Sov. Math. Dokl. 5, 689-692 (1964)

32. Rofe-Beketov, F.S.: Perturbation of a Hill operator having a first moment and nonzero integral creates one discrete level in distant spectral gaps. Mat. Fizika i Funkts. Analiz (Khar'kov) 19, 158-159 (1973) (Russian)

33. Simon. B.: Spectral analysis of rank one perturbations and applications. Lecture given at the 1993 Vancouver Summer School, Proceedings on Mathematical Quantum Theory II: Schrödinger Operators (CRM Proceedings and Lecture Notes). (Feldman, J., Froese, R., Rosen, L.M., eds.). To appear

34. Trubowitz, E.: The inverse problem for periodic potentials. Commun. Pure Appl. Math. 30, 321-337 (1977)

35. Venakides, S.: The infinite period limit of the inverse formalism for periodic potentials. Commun. Pure Appl. Math. 41, 3-17 (1988)

36. Zheludev, V.A.: Eigenvalues of the perturbed Schroedinger operator with a periodic potential. Topics in Mathematical Physics (M.Sh. Birman, ed.), Vol. 2, New York: Consultants Bureau, 1968, pp. 87-101

37. Zheludev, V.A.: Perturbation of the spectrum of the one-dimensional self-adjoint Schrödinger operator with a periodic potential. Topics in Mathematical Physics (M.Sh. Birman, ed.), Vol. 4, New York: Consultants Bureau, 1971, pp. 55-75

Communicated by T. Spencer 
